\title{
Adams operations for bivariant $K$-theory and a filtration using projective lines
}

\author{
Mark E. Walker* \\ University of Nebraska-Lincoln \\ Lincoln, NE 68588-0323
}

May 21, 1997

\begin{abstract}
We establish the existence of Adams operations on the members of a filtration of $K$-theory which is defined using products of projective lines. We also show that this filtration induces the gamma filtration on the rational $K$-groups of a smooth variety over a field of characteristic zero.
\end{abstract}

Key words: $K$-theory, Adams operations, gamma filtration, motivic cohomology

\section{Introduction}

In [8], Grayson studies a filtration on the $K$-theory spectrum of a regular ring $R$ whose $t^{\text {th }}$ stage is defined by considering the category of projective $R$-modules equipped with $t$-tuples of pairwise commuting automorphisms. By a filtration, we mean a sequence of maps of spec-

\footnotetext{
* Partially supported by the National Science Foundation, grant \#DMS-9627754.
} 
$\operatorname{tra}$

$$
\ldots \longrightarrow G W^{t+1}(R) \longrightarrow G W^{t}(R) \longrightarrow \ldots \longrightarrow G W^{0}(R)=K(R)
$$

together with fibration sequences

$$
G W^{t+1}(R) \longrightarrow G W^{t}(R) \longrightarrow G W^{t / t+1}(R)
$$

The filtration studied by Grayson has the additional property that $G W^{t / t+1}(R)$ is the spectrum associated to a chain complex of abelian groups. Grayson's filtration can be extended to a regular scheme $X$, and we let $G W^{t}(X)$ denote the $t^{\text {th }}$ stage of this filtration. Using the long exact sequences of homotopy groups associated to the fibration sequences

$$
G W^{t+1}(X) \longrightarrow G W^{t}(X) \longrightarrow G W^{t / t+1}(X)
$$

one obtains a spectral sequence converging to the $K$-groups of $X$, which one might reasonably hope represents a motivic Atiyah-Hirzebruch spectral sequence, at least for $X$ the spectrum of a regular, local ring.

Using results of Voevodsky, we show in [19] that Grayson's filtration is weakly equivalent to another filtration locally on smooth varieties over perfect fields. This alternate filtration, which was originally suggested by Goodwillie and Lichtenbaum, is the central object of study for this paper. For a regular variety $X$, we let $W^{t}(X)$ denote the $t^{\text {th }}$ stage of this filtration of $K(X)$. Loosely speaking, the definition of 
$W^{t}(X)$ is obtained by replacing the automorphisms in Grayson's filtration, which we think of as arising from the group scheme $\mathbb{G}_{\mathrm{m}}$, with "exomorphisms", which arise from the scheme $\mathbb{P}^{1}$.

More precisely, let $\mathcal{P}(X, Y)$ denote the full subcategory of the category of coherent sheaves on $X \times Y$ consisting of those sheaves $\mathcal{F}$ such that the support of $\mathcal{F}$ maps finitely to $X$ and the pushforward of $\mathcal{F}$ to $X$ is locally free. This is an exact category, and we let $K(X, Y)$ (resp., $\left.K_{q}(X, Y)\right)$ denote the associated $K$-theory spectrum (resp., $K$-group). If $Y=\mathbb{G}_{\mathrm{m}}^{t}:=\mathbb{G}_{\mathrm{m}} \times \cdots \times \mathbb{G}_{\mathrm{m}}$ (with $t$ copies of $\mathbb{G}_{\mathrm{m}}$ ), then $K(X, Y)$ is the so-called $K$-theory of automorphisms. The spectrum $G W^{t}(X)$ of Grayson's filtration is obtained from $K\left(X, \mathbb{G}_{\mathrm{m}}^{t}\right)$ essentially by adding a simplicial direction - i.e., replacing $X$ with the cosimplicial scheme $X \times \Delta^{\bullet}$ - and modding out by certain degeneracies. To define $W^{t}(X)$, we modify in a similar fashion the spectrum $K\left(X, \mathbb{P}^{1} \times \cdots \times \mathbb{P}^{1}\right)$, in which $t$ copies of $\mathbb{P}^{1}$ appear. (One significant difference, however, is that the definition of $G W^{t}(X)$ also involves a $t$-fold delooping, while the definition of $W^{t}(X)$ involves no such delooping.) We describe $W^{t}(X)$ and the map from $W^{t+1}(X)$ to $W^{t}(X)$ explicitly in Section 2 of this paper.

The purpose of this paper is two-fold. First, we give a definition of the Adams operations on $\pi_{q} W^{t}(X)$, for all $q$, which are compatible with the maps induced by the map $W^{t+1}(X) \longrightarrow W^{t}(X)$ and which agree with the Adams operations of Grayson's filtration. For Grayson's 
filtration, the map

$$
G W^{t+1}(X) \longrightarrow G W^{t}(X)
$$

involves the introduction of a loop, which makes the compatibility of the Adams operations very difficult, if not impossible, to show directly. Thus the first main result of this paper has the added benefit of establishing that the Adams operations act compatibly on Grayson's filtration, at least locally on smooth schemes over perfect fields. The second purpose of this paper is to show that the filtration of $K_{q}(X)_{\mathbb{Q}}$ induced by $W^{t}(X)$ is the gamma filtration when $X$ is a smooth variety over a field for which resolution of singularities holds (for example, a field of characteristic zero). These two goals, which seem intimately related, are achieved by very different means, possibly causing confusion for the reader. To clarify the dichotomy, we point out here that although we can describe natural fibration sequences $W^{t}(R) \longrightarrow K(R) \longrightarrow W^{0 / t}(R)$, for certain spectra $W^{0 / t}(R)$, we are unable at this time to establish Adams operations compatible with the associated long exact sequence of homotopy groups. Such a result would allow us to conclude that one has Adams operations on the long exact sequence associated to a certain fibration sequence

$$
W^{t+1}(R) \longrightarrow W^{t}(R) \longrightarrow W^{t / t+1}(R) \text {. }
$$


This fibration sequence is equivalent to the fibration sequence used to define Grayson's spectral sequence, at least for smooth, local rings. If we could establish such compatibility, we would therefore obtain the existence of Adams operations acting on Grayson's spectral sequence compatible with the action on the $K$-groups of $R$. Our inability thus far to establish this compatibility explains the schizophrenic nature of this paper.

Conventions: All schemes in this paper are noetherian and all maps of schemes are separated, unless otherwise indicated.

\section{K-theory background}

In this section we recall and extend some constructions and results from [19] concerning the filtration $W^{t}(X)$ of $K(X)$. As in the introduction, we have the $K$-theory spectrum $K(X, Y)$ associated to schemes $X$ and $Y$. In case $X=\operatorname{Spec} A$ and $Y=\operatorname{Spec} B, K(X, Y)$ is the $K$-theory spectrum of the category of $A$ - $B$ bimodules, which are finitely generated

and projective as $A$-modules. We define $K^{\prime}(X, Y)$ to be the $K$-theory of the analogous category in which the condition that the pushforward be locally free is dropped. 
We will have occasion to know $K(X, Y) \sim K^{\prime}(X, Y)$ for certain special cases. If $X$ is regular, one can easily check (or see [19, Theorem 3.5]) that a sufficient condition for the map to be a weak equivalence is that every closed subscheme of $X \times Y$ which is finite over $X$ is contained in a closed subscheme that is finite and flat over $X$. This property holds when $X$ is quasi-projective over a field $k$ and $Y$ is a product of projective lines. For one easily reduces to the case $Y=\mathbb{P}^{1}$. Now suppose $Z$ is a closed subscheme of $X \times \mathbb{P}^{1}$ which is finite over $X$ and let $\mathcal{I}$ denote the sheaf of ideals defining $Z$. For each point $x$ of $X$, the fiber of $Z \longrightarrow X$ consists of (at most) a finite number of points. This is equivalent to the statement that $\mathcal{I} \otimes \mathcal{O}_{\mathrm{Spec}} k(x) \times \mathbb{P}^{1}$ is non-zero. Since $X$ is quasi-projective and thus has an ample line bundle, we can find an $f \in \Gamma\left(X \times \mathbb{P}^{1}, \mathcal{I}(N, M)\right)$, for $N$ and $M$ sufficiently large, such that $f$ is non-zero in $\mathcal{I}(N, M) \otimes \mathcal{O}_{\text {Spec } k(x) \times \mathbb{P}^{1}}$. (For a sheaf $\mathcal{F}$ on $X \times \mathbb{P}^{1}$, the sheaf $\mathcal{F}(N, M)$ is obtained by tensoring $\mathcal{F}$ with the ample line bundle on $X$ a total of $N$ times and with the usual ample line bundle on $\mathbb{P}^{1}$ a total of $M$ times.) By quasi-compactness, we can find $f_{0}, \ldots, f_{n}$ in $\Gamma\left(X \times \mathbb{P}^{1}, \mathcal{I}(N, M)\right), N$ and $M$ sufficiently large, so that not all the $f_{i}$ 's vanish near any given point of $X$. Let $S$ and $T$ be homogeneous coordinates for $\mathbb{P}^{1}$ and consider $f=f_{0} \cdot S^{A}+f_{1} \cdot S^{A-B} T^{B}+\cdots+f_{n} \cdot T^{A} \in$ $\Gamma\left(X \times \mathbb{P}^{1}, \mathcal{I}(N, M+A)\right)$, where $B$ is a large integer and $A=n B$. For $B>M$, no term of $f_{i} \cdot S^{A-i B} T^{i B}$ can coincide with a term of 
$f_{j} \cdot S^{A-j B} T^{j B}$ if $i \neq j$. Thus $f$ does not vanish at $\operatorname{Spec} k(x) \times \mathbb{P}^{1}$, for any point $x$ of $X$. It follows that $f$ defines a locally principal closed subscheme $D$ of $X \times \mathbb{P}^{1}$ containing $Z$ which is quasi-finite and thus finite over $X$. By the local criterion of flatness, the map $D \longrightarrow X$ is flat.

More generally, we can show $K(X, Y) \sim K^{\prime}(X, Y)$ when $X$ is regular and quasi-projective and $Y$ is a product of projective spaces. Once again, one reduces easily to the case $Y=\mathbb{P}^{n}$. Now consider a closed subscheme $Z$ of $X \times \mathbb{P}^{n}$ which is finite over $X$. Let $f:\left(\mathbb{P}^{1}\right)^{\times n} \longrightarrow \mathbb{P}^{n}$ denote the symmetrization map that identifies $\mathbb{P}^{n}$ with the $n^{\text {th }}$ symmetric product of $\mathbb{P}^{1}$. Observe that $f$ is finite and flat. Let $W$ denote the inverse image of $Z$ under the map $X \times\left(\mathbb{P}^{1}\right)^{\times n} \longrightarrow X \times \mathbb{P}^{n}$. If $\pi_{i}$ denotes the $i^{\text {th }}$ projection $X \times\left(\mathbb{P}^{1}\right)^{\times n} \longrightarrow X \times \mathbb{P}^{1}$, then, as shown previously, $\pi_{i}(W)$ is contained in some $D_{i}$, where $D_{i}$ is a locally principal closed subscheme of $X \times \mathbb{P}^{1}$ finite and flat over $X$. In fact, we can take $D_{i}=D$, for some fixed $D$ which is finite and flat over $X$, so that $W$ is a subset of $D \times_{X} \cdots \times_{X} D$. Further, the image of $D \times_{X} \cdots \times_{X} D$ under id $\times f$ is isomorphic to the relative symmetrization $S^{n}(D / X):=D \times_{X} \cdots \times_{X} D / \Sigma_{n}$, which is easily shown to be finite and flat over $X$ and to contain $Z$.

Let us record these observations with the following lemma. 
LEMMA 2.1. If $Y$ is a product of projective spaces and $X$ is regular and quasi-projective over a field, then the natural map

$$
K(X, Y) \longrightarrow K^{\prime}(X, Y)
$$

is a weak equivalence.

We now define the spectrum $W^{t}(X)$ explicitly. Choose coordinates for the scheme $\mathbb{P}^{1}$ once and for all, and let $\mathbb{P}^{1}$ be pointed by the rational point 1 with coordinates $[1: 1]$. Recall that a $t$-cube of schemes is a functor from the category $\operatorname{Pow}(n)$, defined as the poset of all subsets of $\{1, \ldots, t\}$, to the category of schemes. We write $\mathbb{P}^{\wedge t}$ for the $t$-cube of schemes $I \mapsto \mathbb{P}_{I}^{\wedge t}:=Y_{1} \times \cdots \times Y_{t}$, where $Y_{i}=\mathbb{P}^{1}$ if $i \in I$ and $Y_{i}$ is the closed subscheme consisting of just the point 1 otherwise. In general, the scheme corresponding to a set $I \in \mathcal{P}$ ow $(n)$ of an $n$-cube $C$ is written $C_{I}$. For later use we introduce the exterior product $C \otimes C^{\prime}$ of a $t$-cube $C$ with an $s$-cube $C^{\prime}$. This is by definition the $t+s$-cube defined by identifying $\mathcal{P}$ ow $(t+s)$ with $\mathcal{P}$ ow $(t) \times \mathcal{P}$ ow $(s)$ and sending the pair $I \in \mathcal{P}$ ow $(t), J \in \mathcal{P}$ ow $(s)$ to $C_{I} \times C_{J}^{\prime}$. Thus we may define $\mathbb{P}^{\wedge t}$ to be $\mathbb{P}^{\wedge 1} \otimes \cdots \otimes \mathbb{P}^{\wedge 1}$. Observe that 1 -cube $\mathbb{P}^{\wedge 1}$ admits a splitting given by the structure map $\mathbb{P}^{1} \longrightarrow$ Spec $\mathbb{Z}$, and so the $t$-cube $\mathbb{P}^{\wedge t}$ admits a compatible family of splittings. Let us call such a cube of schemes a split cube. Define $K\left(X, \mathbb{P}^{\wedge t}\right)$ to be the realization of the cube of spectra obtained by applying the functor $K(X,-)$ degreewise to $\mathbb{P}^{\wedge t}$. Finally, 
we define $W^{t}(X)$ to be the geometric realization of the simplicial cube of spectra obtained by applying the functor $K(-,-)$ degreewise to $X \times \Delta^{\bullet}$ in the first variable and $\mathbb{P}^{\wedge t}$ in the second. Recall that $\Delta^{\bullet}$ is the cosimplicial scheme defined by

$$
d \mapsto \operatorname{Spec} \mathbb{Z}\left[x_{0}, \ldots, x_{d}\right] /\left(x_{0}+\cdots+x_{d}-1\right) .
$$

In order to describe the map from $W^{t+1}(X)$ to $W^{t}(X)$ and for other purposes, it is convenient to construct an enlargement of the category of smooth schemes. Fix a ground field $k$, and define $K_{0}(S m / k)$ to be the category whose objects are quasi-projective smooth schemes over $k$ and whose morphisms from $X$ to $Y$ are elements of the abelian group $K_{0}(X, Y)$. Composition of morphisms is given by the pairing

$$
K_{0}(X, Y) \otimes K_{0}(Y, Z) \longrightarrow K_{0}(X, Z)
$$

induced by tensor product. More explicitly, if we define $\pi_{X}: X \times$ $Y \times Z \longrightarrow Y \times Z$ to be the natural projection and define $\pi_{Y}$ and $\pi_{Z}$ similarly, then composition is given by the formula

$$
[\mathcal{F}] \circ[\mathcal{G}]=\left[\pi_{Y *}\left(\pi_{Z}^{*} \mathcal{F} \otimes \pi_{X}^{*} \mathcal{G}\right)\right]
$$

In the affine situation, where $X=\operatorname{Spec} A, Y=\operatorname{Spec} B$, and $Z=$ Spec $C$, composition can be defined more simply as the pairing induced by the bifunctor $-\otimes_{B}-$. We observe that $K_{0}(S m / k)$ is an additive category provided the empty scheme is allowed as an object. 
Taking the structure sheaf associated to the graph of a map of schemes from $X$ to $Y$ yields a functor

$$
S m / k \longrightarrow K_{0}(S m / k)
$$

We will regard maps of schemes as giving maps in $K_{0}(S m / k)$ without comment. There are also external pairings

$$
K_{0}(X, Y) \otimes K_{0}(Z, W) \longrightarrow K_{0}(X \times Z, Y \times Z)
$$

induced by pulling back under the evident projections and taking tensor product. This pairing should be thought of as defining the tensor product of morphisms, where we define the tensor product of two schemes $X$ and $Z$ to be $X \times Z$. More generally, we define $K_{0}(S c h / \mathbb{Z})$ to be the category with objects all (separated, noetherian) schemes and morphisms given by the abelian groups $K_{0}(X, Y)$.

The group $K_{q}(X, Y)$ is a bifunctor (contravariant in the first variable and covariant in the second) from $K_{0}(S m / k)$ to abelian groups, and, more generally, $K(X, Y)$ is a bifunctor "up to homotopy" with values in the category of spectra. Observe that $K_{0}(X, \operatorname{Spec} k) \cong K_{0}(X)$. In particular, elements of $K_{0}(Y)$ define natural maps $K(X, Y \times Z) \longrightarrow$ $K(X, Z)$, by using composition and tensoring by the identity map on $Z$. We let $\partial_{k}$ denote the element of $K_{0}\left(\mathbb{P}^{k}\right)$ which is the class of a hyperplane; i.e., $\partial_{k}=1-[\mathcal{O}(-1)]$. Abusing notation, we also let $\partial_{k}$ 
denote both the map from $K\left(X, Y \times \mathbb{P}^{k}\right)$ to $K(X, Y)$ and the associated map on homotopy groups induced by $\partial_{k}$.

The map from $W^{t+1}(X) \longrightarrow W^{t}(X)$ is induced by applying $\partial_{1}$ to the last copy of $\mathbb{P}^{1}$ in the definition of $W^{t+1}(X)$. Since the composition in $K_{0}(S m / k)$

$$
\operatorname{Spec} k \stackrel{1}{\longrightarrow} \mathbb{P}^{1} \stackrel{\partial_{1}}{\longrightarrow} \operatorname{Spec} k
$$

is zero, this gives a well-defined map between cubes of spectra, if we regard the target cube as being $t+1$-dimensional by adding one-point spectra as needed. Actually, to get this the be a strictly commuting map of cubes of spectra (not just up to homotopy) requires some care, but can be accomplished.

Observe that $W^{0}(X) \sim K(X)$ for $X$ regular, so that we have a filtration

$$
\ldots \longrightarrow W^{t+1}(X) \longrightarrow W^{t}(X) \longrightarrow \ldots \longrightarrow K(X)
$$

In Section 7 we will describe fibration sequences

$$
W^{t+1}(X) \longrightarrow W^{t}(X) \longrightarrow W^{t / t+1}(X)
$$

for certain spectra $W^{t / t+1}(X)$. The associated long exact sequences of homotopy groups give a spectral sequence converging to the $K$-groups of $X$, which is a good candidate for the motivic "Atiyah-Hirzebruch" spectral sequence for $X$ regular (but not necessarily local). 


\section{3. $\mathcal{M}$-schemes}

In this section, we study exterior power operations in a rather general bivariant setting. The objects of interest here are graded commutative scheme monoids. By this we mean a scheme $M$ which is a disjoint union of schemes

$$
M=M_{0} \amalg M_{1} \amalg \ldots
$$

together with a pairing $\mu: M \times M \longrightarrow M$, which restricts to maps $M_{i} \times M_{j} \longrightarrow M_{i+j}$, and a unit $e: \operatorname{Spec} Z \longrightarrow M_{0}$ which make $M$ into a commutative monoid object in the category of schemes. We also require that the schemes $M_{i}$ are noetherian (and separated). Observe that $M$ is therefore locally noetherian. Let us call such a scheme an "M-scheme". We will also consider the relative case in which $M$ is a scheme over $X$, the unit of $M$ is a map $e: X \longrightarrow M$, and multiplication in $M$ is a map $M \times_{X} M \longrightarrow M$. We call such a scheme an " $\mathcal{M}$-scheme over $X$ ". Observe that given an $\mathcal{M}$-scheme $M$, the scheme $X \times M$ is an $\mathcal{M}$-scheme over $X$.

A noetherian commutative monoid scheme $M$ equipped with the trivial grading $M_{0}=M$ and $M_{i}=\emptyset$ for $i>0$ is an example of an $\mathcal{M}$ scheme. We will have occasion to regard $\mathbb{G}_{\mathrm{m}}^{1}$ and $\mathbb{A}^{1}$ (with product given by multiplication) as $\mathcal{M}$-schemes in this manner. Another important example is constructed from any quasi-projective scheme $Y$ by letting 
$M=\coprod_{n} \mathrm{~S}^{n} Y$, where $\mathrm{S}^{n} Y$ is the $n^{\text {th }}$ symmetric power of $Y$ formed by taking the quotient of $Y \times \cdots \times Y$ by the action of the symmetric group on $n$ symbols. Let us call this $\mathcal{M}$-scheme $S \cdot Y$. If $Y$ happens to be a commutative monoid scheme, there is a natural map $S \cdot Y \longrightarrow$ $Y$. If $Y \longrightarrow X$ is a map of schemes, then we define $\mathrm{S}^{n}(Y / X)$ to be $Y \times_{X} \cdots \times_{X} Y / \Sigma_{n}$. The scheme $S^{\cdot}(Y / X):=\coprod_{n} \mathrm{~S}^{n}(Y / X)$ is an $\mathcal{M}$ scheme over $X$.

We will be especially interested in the $\mathcal{M}$-scheme $S \cdot \mathbb{P}^{1}$. Recall that $\mathrm{S}^{n} \mathbb{P}^{1} \cong \mathbb{P}^{n}$, for all $n$. The scheme $\mathrm{S}^{n} \mathbb{P}^{1}$ parametrizes effective zero cycles of degree $n$ on $\mathbb{P}^{1}$ and the product structure corresponds to addition of cycles. We can generalize this example to an arbitrary projective scheme $Y$ by considering the Chow monoid of effective $r$-cycles on $Y$, which Friedlander and Lawson write as

$$
\mathcal{C}_{r}(Y)=\coprod_{d} C_{r, d}(Y)
$$

where $C_{r, d}(Y)$ is the projective variety parametrizing effective $r$-cycles of degree $d$ on $Y$.

If $M$ and $N$ are two $\mathcal{M}$-schemes, we want to define their product by $(M \times N)_{i}=M_{i} \times N_{i}$, which will also be an $\mathcal{M}$-scheme. (We could introduce multigraded monoid schemes instead, but we have no need for this generality.) 
We now consider the category $\mathcal{P}(X, M)$, where $M$ is an $\mathcal{M}$-scheme. This is actually a special case of the category $\mathcal{P}(M / X)$ consisting of coherent sheaves $\mathcal{F}$ on $M$ whose support is finite over $X$ and such that the pushforward of $\mathcal{F}$ to $X$ is locally free, where $M$ is an $\mathcal{M}$ scheme over $X$. In fact, we have $\mathcal{P}(X, M)=\mathcal{P}(X \times M / X)$. The first observation to make is that due to the noetherianess of $X$, an object of $\mathcal{P}(X / M)$ is supported on $M_{0} \amalg \cdots \amalg M_{t}$ for $t$ sufficiently large. Thus $K(M / X)=\bigoplus_{n} K\left(M_{n} / X\right)$ as spectra.

There is a biexact functor

$$
-\otimes-: \mathcal{P}(M / X) \times \mathcal{P}(M / X) \longrightarrow \mathcal{P}(M / X)
$$

obtained as follows. Given $\mathcal{F}$ and $\mathcal{G}$ objects of $\mathcal{P}(M / X)$, their external tensor product $\mathcal{F} \otimes \mathcal{G}$, which is a coherent sheaf on $M \times_{X} M$, is defined by $\pi_{1}^{*} \mathcal{F} \otimes \pi_{2}^{*} \mathcal{G}$, where $\pi_{i}: M \times_{X} M \longrightarrow M$ is the $i^{\text {th }}$ projection map. We can now use the multiplication in $M$ to define $\mathcal{F} \otimes \mathcal{G}:=\mu_{*}(\mathcal{F} \otimes \mathcal{G})$. The support of $\mathcal{F} \otimes \mathcal{G}$ is finite over the support of $\mathcal{F}$ (and the support of $\mathcal{G}$ ) and thus is finite over $X$. Additionally, if $p: M \times_{X} M \longrightarrow X$ and $q: M \longrightarrow X$ are the evident structure maps, then

$$
\begin{aligned}
p_{*}(\mathcal{F} \otimes \mathcal{G}) & \cong q_{*}\left(\mathcal{F} \otimes\left(\pi_{1}\right)_{*} \pi_{2}^{*} \mathcal{G}\right) \\
& \cong q_{*}\left(\mathcal{F} \otimes q^{*} q_{*} \mathcal{G}\right) \\
& \cong q_{*} \mathcal{F} \otimes q_{*} \mathcal{G}
\end{aligned}
$$


which shows us that $\mathcal{F} \otimes \mathcal{G}$ belongs to $\mathcal{P}\left(M \times_{X} M / X\right)$. It follows $\mathcal{F} \otimes \mathcal{G}$ is an object of $\mathcal{P}(M / X)$. The biexactness of $-\otimes-$ in this context is established by similar computations.

We also have a notion of exterior power for objects of $\mathcal{P}(M / X)$. We use the following construction of Grayson found in [6]. Given $\mathcal{F}$ in $\mathcal{P}(M / X)$, let $Z$ be a closed subscheme of $M$ finite over $X$ on which $\mathcal{F}$ is supported. Then $\mathcal{F}$ may be regarded as an object of $\mathcal{P}(Z / X)$, and we will first consider exterior powers on this category. Consider the $\mathcal{M}$-scheme $S^{\cdot}(Z / X)$ over $X$. Its $n^{\text {th }}$ graded piece is $Z_{n}:=\mathrm{S}^{n}(Z / X)$. Since $Z$ is finite over $X, Z$ is defined by a coherent sheaf $\mathcal{A}$ of $\mathcal{O}_{X^{-}}$ algebras. Further the scheme $Z_{n}$ is finite over $X$ and is defined by the coherent sheaf $\mathcal{A}_{n}:=\left(\mathcal{A} \otimes_{\mathcal{O}_{X}} \cdots \otimes_{\mathcal{O}_{X}} \mathcal{A}\right)^{\Sigma_{n}}$ of $\mathcal{O}_{X}$-algebras, where the superscript $\Sigma_{n}$ denotes taking invariants. An object of $\mathcal{P}\left(Z_{n} / X\right)$ is a locally free sheaf $\mathcal{N}$ on $X$ which is also a $\mathcal{A}_{n}$-module in the sense that there is a map

$$
\mathcal{A}_{n} \longrightarrow \operatorname{End}_{\mathcal{O}_{X}}(\mathcal{N})
$$

of $\mathcal{O}_{X}$-algebras. Using this description of objects of $\mathcal{P}\left(Z_{n} / X\right)$, the tensor product defined above for an arbitrary $\mathcal{M}$-scheme over $X$ is the pairing

$$
\mathcal{P}\left(Z_{n} / X\right) \times \mathcal{P}\left(Z_{m} / X\right) \longrightarrow \mathcal{P}\left(Z_{n+m} / X\right)
$$


given by $(\mathcal{N}, \mathcal{M}) \mapsto \mathcal{N} \otimes_{X} \mathcal{M}$, where the sheaf $\mathcal{N} \otimes_{X} \mathcal{M}$ determines an object of $\mathcal{P}\left(Z_{n+m} / X\right)$ using the composit map

$$
\mathcal{A}_{n+m} \hookrightarrow \mathcal{A}_{n} \otimes_{X} \mathcal{A}_{m} \longrightarrow \operatorname{End}_{X}\left(\mathcal{N} \otimes_{X} \mathcal{M}\right)
$$

We define exterior powers in this context as the functor

$$
\Lambda_{X}^{k}: \mathcal{P}\left(Z_{n} / X\right) \longrightarrow \mathcal{P}\left(Z_{n k} / X\right)
$$

given by taking the $k^{\text {th }}$ exterior power of $\mathcal{N}$ as an $\mathcal{O}_{X}$-module and defining the structure as an $\mathcal{A}_{n k}$-module using the composit map

$$
\mathcal{A}_{n k} \hookrightarrow\left(\mathcal{A}_{n} \otimes_{X} \cdots \otimes_{X} \mathcal{A}_{n}\right)^{\Sigma_{k}} \longrightarrow \operatorname{End}_{X}\left(\Lambda^{k} \mathcal{N}\right)
$$

We can now describe the exterior power functor on $\mathcal{P}(M / X)$. Observe there is a natural functor $G_{n}: \mathcal{P}\left(Z_{n} / X\right) \longrightarrow \mathcal{P}(M / X)$ because $M$ is a commutative monoid. We define $\Lambda_{X}^{k} \mathcal{F}$ for $\mathcal{F}$ an object of $\mathcal{P}(M / X)$ supported on $Z$ to be the image under $G_{n}$ of the $k^{\text {th }}$ exterior power of $\mathcal{F}$ as an object of $\mathcal{P}(Z / X)$. Up to a natural isomorphism, the construction is independent of the choice of $Z$.

Observe that $\Lambda_{X}^{k}$ is a degree $k$ functor in the sense that it restricts to a functor

$$
\Lambda_{X}^{k}: \mathcal{P}\left(M_{n} / X\right) \longrightarrow \mathcal{P}\left(M_{n k} / X\right)
$$

Simlarly the notion of tensor product we have defined restricts to a functor

$$
\mathcal{P}\left(M_{i} / X\right) \times \mathcal{P}\left(M_{j} / X\right) \longrightarrow \mathcal{P}\left(M_{i+j} / X\right)
$$


So in fact we have notions of tensor product and exterior power on the family of categories $\mathcal{P}\left(M_{n} / X\right)$ indexed by the nonnegative integers. If we regard $\mathcal{P}(M / X)$ as a constant family of categories, then the inclusions $\mathcal{P}\left(M_{n} / X\right) \longrightarrow \mathcal{P}(M / X)$ combine to give a map of families of categories which is compatible with tensor product and exterior power up to a natrual isomorphism.

We now recall Grayson's notion of an exact category equipped with exterior powers. We refer the reader to [7] for a detailed list of axioms and properties. Most generally, an exact category with exterior powers is a family of exact categories $\mathcal{P}_{n}$, for $n \geq 0$, with a biexact tensor product $\mathcal{P}_{i} \times \mathcal{P}_{j} \longrightarrow \mathcal{P}_{i+j}$ and "exterior power" functors

$$
F_{k} \mathcal{P}_{n} \longrightarrow \mathcal{P}_{n k}
$$

where, given an exact category $\mathcal{C}, F_{k} \mathcal{C}$ is the category of chains of admissible monomorphisms of length $k$ in $\mathcal{C}$. The functors are required to satisfy a lengthly list of axioms, all of which are more or less evident in the primordial case of the category of projective $R$-modules (regarded as a constant family of exact categories). In our context, the required functor $F_{k} \mathcal{P}(M / X) \longrightarrow \mathcal{P}(M / X)$ is defined by sending $\mathcal{F}_{1} \hookrightarrow \cdots \hookrightarrow$ $\mathcal{F}_{k}$ to the image of the map

$$
\mathcal{F}_{1} \otimes \cdots \otimes \mathcal{F}_{k} \longrightarrow \Lambda^{k} \mathcal{F}_{k}
$$


This definition is analogous to the corresponding definition for the category of projective $R$-modules. To see that the map (1) exists, it suffices to consider the special case $M=S^{\cdot}(Z / X)$, for $Z$ finite over $X$, and in this case the definition of the map is evident.

THEOREM 3.1. For an $\mathcal{M}$-scheme $M$ over $X$, the category $\mathcal{P}(M / X)$ equipped with the notions of tensor product and exertior power defined above satisfies Grayson's axioms (i.e., axioms (E1)-(E5) of [7, §7]) for a category with exerior powers. Furthermore, the family of categories $\mathcal{P}\left(M_{n} / X\right)$ satisfies Grayson's axioms for a family of categories with exterior powers, and the functor $\mathcal{P}\left(M_{n} / X\right) \longrightarrow \mathcal{P}(M / X)$ is a functor of such categories.

Proof. Every finite collection of morphisms of $\mathcal{P}(M / X)$ are realized in the category

$$
\mathcal{P}(S \cdot(Z / X) / X)
$$

for some closed subset $Z$ of $M$ which is finite over $X$. Checking Grayson's axioms, one sees that it suffices to consider the special case $M=$ $S \cdot(Z / X)$ for such a $Z$. In fact, it suffices to show the family of categories $\mathcal{P}\left(\mathrm{S}^{n}(Z / X) / X\right)$ satisfies the axioms.

Let $Z_{n}=\mathrm{S}^{n}(Z / X)$. Recall $Z$ corresponds to a coherent $\mathcal{O}_{X}$-algebra $\mathcal{A}$ and $Z_{n}$ corresponds to the coherent $\mathcal{O}_{X}$-algebra $\mathcal{A}_{n}$ of $\Sigma_{n}$-invariant as before. Objects of $\mathcal{P}\left(Z_{n} / X\right)$ correspond to locally free $\mathcal{O}_{X}$-modules 
equipped with $\mathcal{A}_{n}$-actions. So objects of $\mathcal{P}\left(Z_{n} / X\right)$ are essentially objects in the category $\mathcal{P}(X)$ of locally free $\mathcal{O}_{X}$-modules equipped with extra structure, and moreover tensor product and exterior powers are defined in terms of the corresponding functors on $\mathcal{P}(X)$. The various maps and exact sequences required in Grayson's axioms are now straigforward to verify, since these axioms hold for the category $\mathcal{P}(X)$.

We would like to be able to consider functors of exact categories with exterior powers. To avoid an explosion of axioms, we define such a functor to be one that strictly commutes with tensor product and exerior powers. That is, a functor $\mathcal{P}_{*} \longrightarrow \mathcal{Q}_{*}$ of families of exact categories with exterior powers is an exact functor such that the square of functors

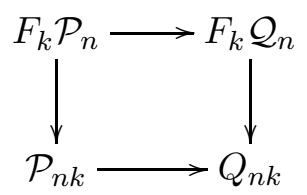

commutes and a similar statement holds for tensor products.

The functorality of $\mathcal{P}(X, M)$ does not meet this stingent requirement as currently configured. To rectify this, we must replace $\mathcal{P}(X, M)$ by an equivalent category with more structure, which is a slight generalization of a constructin of Grayson found in $[8, \S 10]$. As a first step, take both $X$ and $M$ to be affine so that we are considering the category $\mathcal{P}(A, B)$ of $A$ - $B$-bimodules which are projective of finite rank over $A$, where $B$ is 
a cocommutative comonoid object. We define a new category $\mathcal{P}^{\prime}(A, B)$ in which an object consists of (1) an assignment $\alpha: f \mapsto P_{f}$, where $f: A \longrightarrow A_{1}$ is a ring map and $P_{f}$ is an object of $\mathcal{P}\left(A_{1}, B\right)$, and (2) an assigment $\beta$ which sends a map

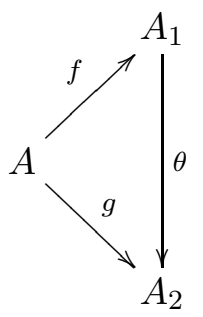

of $A$-algebras to an $A_{1}$-linear map $\beta(\theta): P_{f} \longrightarrow P_{g}$ such that the induced map $P_{f} \otimes_{A_{1}} A_{2} \longrightarrow P_{g}$ is an isomorphism. The assignment $\beta$ is also required to satisfy $\beta\left(\operatorname{id}_{A_{1}}\right)=\mathrm{id}$ and $\beta(\theta \circ \phi)=\beta(\phi) \circ \beta(\theta)$. Morphisms in $\mathcal{P}^{\prime}(A, B)$ are the evident natural transformations. Then $\mathcal{P}^{\prime}(A, B)$ is equivalent to $\mathcal{P}(A, B)$ via the functor which sends $(\alpha, \beta)$ to $\alpha\left(\operatorname{id}_{A}\right)$. Further, $\mathcal{P}(A, B)$ is an exact category with exterior powers in which tensor product and exterior power are defined degreewise over the collection of $A$-algebras. The equivalence $\mathcal{P}^{\prime}(A, B) \longrightarrow \mathcal{P}(A, B)$ is an equivalence of such categories.

Given a ring map $A \longrightarrow C$, the functor $\mathcal{P}^{\prime}(A, B) \longrightarrow \mathcal{P}^{\prime}(C, B)$ is defined by restricting the domains of $\alpha$ and $\beta$, and thus $A \mapsto \mathcal{P}^{\prime}(A, B)$ is a functor from the category of commutative rings to the category of exact categories. In fact, it is a functor from rings to the category of exact categories with exterior powers, in that in commutes with 
tensor product and exterior powers strictly. Given a map $D \longrightarrow B$ of cocommutative comonoid objects in the category of rings, the functor $\mathcal{P}^{\prime}(A, B) \longrightarrow \mathcal{P}^{\prime}(A, D)$ is given by restriction of scalars, and thus clearly defines a functor from such rings to exact categories with exterior powers.

Thus, we can assume $\mathcal{P}(X, M)$ is strictly functorial when both $X$ and $M$ are affine. The reader who is convinced by the affine case that such strict functorality is possible more generally may skip the following few paragraphs in which we extend the preceeding considerations to the non-affine case in all its gory detail. Fix $X$ a scheme and $M$ an $\mathcal{M}$ scheme and let $\mathcal{C}$ be the product of the category of schemes over $X$ (i.e., maps $f: Y \longrightarrow X$ ) with the category of maps $p: M \longrightarrow N$ of $\mathcal{M}$-schemes with source $M$. Define a category $\mathcal{P}^{\prime}(X, M)$ for which an object consists of an assigment

$$
\alpha:(f, p) \mapsto \mathcal{F}_{f, p}
$$

where $\mathcal{F}_{f, p}$ is an object of $\mathcal{P}(Y, N)$, and an assigment $\beta$ which we describe as follows. A morphism of $\mathcal{C}$ from $(f, p)$ to $(g, q)$ is a pair 
of commuting triangles
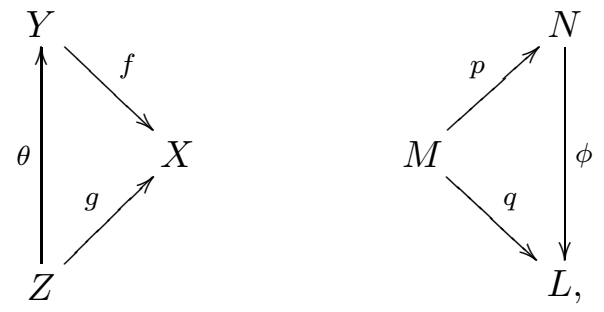

which we write as $(\theta, \phi)$. The assigment $\beta$ associates to each such $(\theta, \phi)$ a commuting square of maps

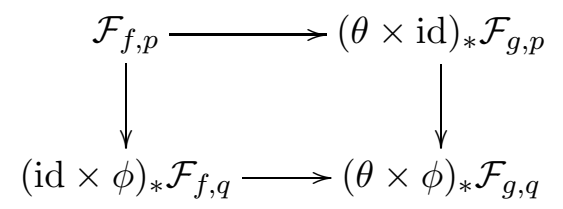

such that the vertical maps are isomorphisms and the horizontal maps correspond to isomorphisms under the evident adjointness correspondences. Additionally, the assignment $\beta$ is required to satisfy the following conditions: (1) if $\theta=\mathrm{id}$, then the horizontal arrows in (2) are equalities; (2) if $\phi=\mathrm{id}$, then the vertical arrows in (2) are equalities; and (3) given composable morphisms $\left(\theta^{\prime}, \phi^{\prime}\right)$ and $(\theta, \phi)$ in $\mathcal{C}$, which are by definition morphisms fitting into a commutative diagram such as
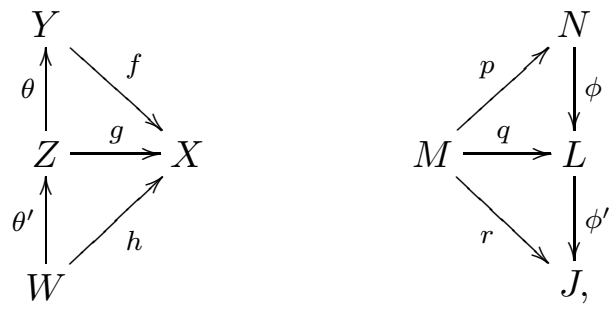
then "everything commutes". An example of the commutativity required in condition (3) is that the diagram

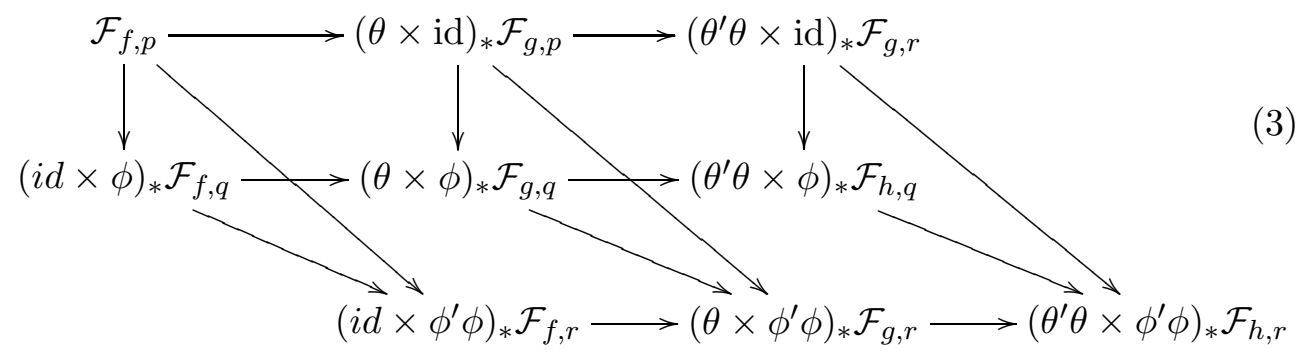

must commute. In fact, this diagram and the similar one obtained by considering the map $\mathcal{F}_{f, p} \longrightarrow\left(\theta^{\prime} \theta \times \mathrm{id}\right)_{*} \mathcal{F}_{g, r}$ encapsulate all of the commutativity required. The morphisms appearing in (3) which are not part of the data defining $\beta$ are natural constructions using this data. For example, the map from $(\theta \times \mathrm{id})_{*} \mathcal{F}_{g, p} \longrightarrow\left(\theta^{\prime} \theta \times \mathrm{id}\right)_{*} \mathcal{F}_{h, p}$ is constructed by applying $\left(\theta^{\prime} \times \mathrm{id}\right)_{*}$ to a map which is part of the data defining $\beta$ and then using the natural transformation $\theta_{*}^{\prime}\left(\theta_{*} \mathcal{F}\right) \longrightarrow\left(\theta^{\prime} \theta\right)_{*} \mathcal{F}$.

There is a functor from $\mathcal{P}^{\prime}(X, M)$ to $\mathcal{P}(X, M)$ given by $(\alpha, \beta) \mapsto$ $\alpha(\mathrm{id}, \mathrm{id})$. This functor is an equivalence of categories, with inverse given by sending $\mathcal{F}$ to the assignments $\alpha:(f, p) \mapsto f^{*}\left(p_{*} \mathcal{F}\right)$ and $\beta$, which is defined using a plethora of natural equivalences. Given a morphism $X^{\prime} \longrightarrow X$, the functor $\mathcal{P}^{\prime}\left(X^{\prime}, M\right)$ is essentially given by restricting the domains of $\alpha$ and $\beta$. The same can be said for a functor $\mathcal{P}^{\prime}(X, M) \longrightarrow$ $\mathcal{P}^{\prime}\left(X, M^{\prime}\right)$. In particular, the map $(X, M) \mapsto \mathcal{P}^{\prime}(X, M)$ is actually a functor in the evident sense. 
What is more, we may define a tensor structure on $\mathcal{P}^{\prime}(X, M)$ by doing so degreewise with respect to $\alpha$. Thus, if $(\alpha, \beta)$ is one object, where we let $\alpha(f, p)=\mathcal{F}_{f, p}$, and $\left(\alpha^{\prime}, \beta^{\prime}\right)$ is another, where $\alpha^{\prime}(f, p)=$ $\mathcal{G}_{f, p}$, then $(\alpha, \beta) \otimes\left(\alpha^{\prime}, \beta^{\prime}\right)$ is given by the assignments $\alpha \otimes \alpha^{\prime}:(f, p) \mapsto$ $\mathcal{F}_{f, p} \otimes \mathcal{G}_{f, p}$ and $\beta \otimes \beta^{\prime}$, which is defined using natural transformations

of the form $\rho_{*} \mathcal{F} \otimes \rho_{*} \mathcal{G} \longrightarrow \rho_{*}(\mathcal{F} \otimes \mathcal{G})$ repeatedly. Verification that this tensor product actually gives an object of $\mathcal{P}^{\prime}(X, M)$ is a tedious but straightforward process. The exterior power functors are defined on $\mathcal{P}^{\prime}(X, M)$ in a similar degreewise fashion. Ultimately, one sees that $\mathcal{P}^{\prime}(X, M)$ is strictly functorial on the category of pairs $(X, M)$ with values in the category of (families of) exact categories with exterior powers, and that $\mathcal{P}^{\prime}(X, M) \longrightarrow \mathcal{P}(X, M)$ is an equivalence of categories with exterior powers.

\section{Some connections to motivic cohomology}

Take $X$ and $Y$ to be quasi-projective schemes over a field $k$, and assume $X$ is integral for simplicity. The $k^{\text {th }}$ exterior power functor induces the map $K_{0}(X, Y) \longrightarrow K_{0}\left(X, \mathrm{~S}^{k} Y\right)$. We can define a determinant function from the isomorphism class of objects of $\mathcal{P}(X, Y)$ to the isomorphism classes of the objects of $\mathcal{P}(X, S \cdot Y)$ by taking the highest nonzero exte- 
rior power of objects of $\mathcal{P}(X, Y)$. The determinant of a rank $d$ object $\mathcal{F}$ is a rank one object of $\mathcal{P}\left(X, \mathrm{~S}^{d} Y\right)$, where rank is defined as rank as an $\mathcal{O}_{X}$-modules after pushforward. Observe that the support of a rank one object maps isomorphically to $X$. Thus, to specify a rank one object in $\mathcal{P}\left(X, \mathrm{~S}^{n} Y\right)$ amounts, up to isomorphism, to choosing a line bundle on $X$ and a map $X \longrightarrow \mathrm{S}^{n} Y$. Thus if $\operatorname{Pic}\left(X, \mathrm{~S}^{n} Y\right)$ denotes the collection of isomorphism classes of rank one objects in $\mathcal{P}\left(X, \mathrm{~S}^{n} Y\right)$, then we have $\operatorname{Pic}\left(X, \mathrm{~S}^{n} Y\right) \cong \operatorname{Pic}(X) \times \operatorname{Hom}\left(X, \mathrm{~S}^{n} Y\right)$. Further, tensor product of rank one objects gives a pairing

$$
\operatorname{Pic}\left(X, \mathrm{~S}^{n} Y\right) \times \operatorname{Pic}\left(X, \mathrm{~S}^{m} Y\right) \longrightarrow \operatorname{Pic}\left(X, \mathrm{~S}^{n+m} Y\right)
$$

which corresponds to the product on $\operatorname{Pic}(X) \times \operatorname{Hom}(X, S \cdot Y)$ given by the abelian groups structure on $\operatorname{Pic}(X)$ and the monoid structure of $S \cdot Y$. We let $\operatorname{Hom}(X, S \cdot Y)^{+}$denote the group completion of the commutative monoid $\operatorname{Hom}\left(X, S^{\cdot}\right)$. We obtain by the universal property of $K_{0}$ a homomorphism

$$
\operatorname{det}: K_{0}(X, Y) \longrightarrow \operatorname{Pic}(X) \times \operatorname{Hom}(X, S \cdot Y)^{+} \text {. }
$$

If $M$ is an $\mathcal{M}$-scheme, we can use its multiplication to get the map

$$
\operatorname{det}: K_{0}(X, M) \longrightarrow \operatorname{Pic}(X) \times \operatorname{Hom}(X, M)^{+} \text {. }
$$

Define $C_{0}(X, Z)$ to be the free abelian group on the set of closed, integral subschemes of $X \times Z$ which are finite and dominant over $X$. 
Suslin and Voevodsky [16, Theorem 6.8] show that for any scheme $Z$ over $k$ and any normal connected scheme $X$ over $k$, one has an isomorphism

$$
C_{0}(X, Z)[1 / p] \cong \operatorname{Hom}(X, S \cdot Z)[1 / p]^{+},
$$

where $p$ is the exponential characteristic of $k$. In case $X$ is regular and $Z$ is sufficiently nice, we have that $K_{0}^{\prime}(X, Z) \cong K_{0}(X, Z)$. In this situation, the map inducing the equivalence (4) can be described as the composite

$$
\begin{aligned}
C_{0}(X, Z) \longrightarrow K_{0}^{\prime}(X, Z) & \cong K_{0}(X, Z) \stackrel{\operatorname{det}}{\longrightarrow} \\
& \operatorname{Pic}(X) \times \operatorname{Hom}(X, S \cdot Z))^{+} \longrightarrow \operatorname{Hom}(X, S \cdot Z)^{+},
\end{aligned}
$$

where the first map takes a closed integral subscheme of $X \times Z$ to the class of its structure sheaf.

Additionally, consider the monoid $\mathrm{S}^{d}\left(\left(\mathbb{P}^{1}\right)^{\times t}\right)$, and the natural map

$$
\begin{aligned}
K\left(X,\left(\mathbb{P}^{1}\right)^{\times t}\right) & \longrightarrow K_{0}\left(X,\left(\mathbb{P}^{1}\right)^{\times t}\right) \\
& \longrightarrow \operatorname{Pic}(X) \times \operatorname{Hom}\left(X, S^{\cdot}\left(\left(\mathbb{P}^{1}\right)^{\times t}\right)\right)^{+} \longrightarrow \operatorname{Hom}\left(X, S^{\cdot}\left(\left(\mathbb{P}^{1}\right)^{\times t}\right)\right)^{+}
\end{aligned}
$$

Using the equivalence (4), replacing $X$ with the cosimplicial scheme $X \times \Delta^{\bullet}$, and using the cube $\mathbb{P}^{\wedge t}$ of schemes, we get a natural map

$$
W^{t}(X)=K\left(X \times \Delta^{\bullet}, \mathbb{P}^{\wedge t}\right) \longrightarrow C_{0}\left(X \times \Delta^{\bullet}, \mathbb{P}^{\wedge t}\right)
$$


This latter chain complex is (equivalent to) Voevodsky's proposed definition of the weight $t$ motivic complex for a smooth variety $X$ (see $[18])$.

CONJECTURE 4.1. For $X$ a smooth variety over $k$, the sequence

$$
W^{t+1}(X) \longrightarrow W^{t}(X) \longrightarrow C_{0}\left(X \times \Delta^{\bullet}, \mathbb{P}^{\wedge t}\right)
$$

is a fibration sequence of spectra (possibly only after inverting $p>0$ if char $k=p$ ), and thus there is a spectral sequence whose $E_{2}$-terms are Voevodsky's motivic cohomology groups of $X$ and which converges to the $K$-groups of $X$.

Remark 4.2. One can easily show that the composite in (5) is homotopic to the zero map. For observe that it factors through the geometric realization of the composition of the maps of simplicial abelian groups

$$
K_{0}\left(X \times \Delta^{\bullet}, \mathbb{P}^{\wedge t+1}\right) \longrightarrow K_{0}\left(X \times \Delta^{\bullet}, \mathbb{P}^{\wedge t}\right) \longrightarrow C_{0}\left(X \times \Delta^{\bullet}, \mathbb{P}^{\wedge t}\right) .
$$

But for any $X$ and $Y$, one can check that the composite map

$$
K_{0}\left(X, Y \times \mathbb{P}^{1}\right) \stackrel{\partial_{1}}{\longrightarrow} K_{0}(X, Y) \longrightarrow \operatorname{Hom}(X, S \cdot Y)^{+}
$$

is zero. 


\section{Lambda and Adams operations on $K(X, M)$}

Since we have addressed the nagging functorality questions in Section

3 , we can without worry regard $\mathcal{P}(X, M)$ as an exact category with exterior powers, natural in both $X$ and $M$. Given any family of exact categories with exterior power operations $\mathcal{P}_{n}$, Grayson defines certain homotopy classes of maps

$$
\lambda^{k}: K\left(\mathcal{P}_{n}\right) \longrightarrow K\left(\mathcal{P}_{n k}\right)
$$

on $K$-theory spaces. In case $\mathcal{P}$ is the category of projective $R$-modules, these map are known to induce the usual $\lambda$-operations on the $K$-groups of $R$. To be precise, the map $\lambda^{k}$ is defined $[7, \S 7]$ as a map of multisimplicial sets:

$$
\lambda^{k}: \operatorname{Sub}_{k} \text { G. } \mathcal{P}_{n} \longrightarrow \text { G. }{ }^{k} \mathcal{P}_{n k} .
$$

The symbol $\mathrm{Sub}_{k}$ denotes a type of barycentric subdivision functor which associates to any simplicial set $Y$ a $k$-fold multisimplicial set $\mathrm{Sub}_{k}$ whose geometric realization is naturally homeomorphic to that of $Y$ via a map which we write as

$$
\theta^{k}:\left|\operatorname{Sub}^{k} Y\right| \longrightarrow|Y|
$$

(see $[7, \S 4]$ ). The functor $\mathrm{G}$. is the $G$-construction of $K$-theory and

G. ${ }^{k}$ denotes the $G$-construction applies $k$-times to a category to form 
a $k$-fold multisimplicial set. There is a natural homotopy equivalence $\mid$ G. $\mathcal{M}|\stackrel{\sim}{\longrightarrow}|$ G. $^{k} \mathcal{M} \mid$. (See [5] for details.) For our purposes, it will be most convenient to define $\lambda^{k}$ to be the map of spaces from $|\mathrm{G} . \mathcal{P}|$ to $\left|\mathrm{G} .{ }^{k} \mathcal{P}\right|$ defined as the composite of $\left(\theta^{k}\right)^{-1}$ with the realization of the map of (6).

Since we require a functor of exact categories with exterior powers to respect the structures in such a strong sense, it is clear that given such a functor $\mathcal{P} \longrightarrow \mathcal{Q}$ (or such a functor of families of categories), the operation $\lambda^{k}$ fits into the square

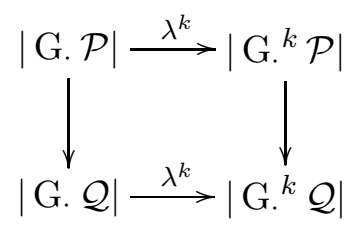

which commutes strictly.

For a arbitrary $\lambda$-ring $A$, it is customary to define Adams operations via the equation

$$
\Psi_{t}(x):=\sum_{k} \Psi^{k}(x) t^{k}=-t \frac{d}{d t} \lambda_{t}(x) .
$$

In general, the $k^{\text {th }}$ Adams operation will be an endomorphism of $A$ as an abelian group, but not necessarily as a ring unless $A$ is a special $\lambda$-ring. The equation (7) is equivalent to the system of equations

$$
\Psi^{k}(x)=P_{k}\left(\lambda^{1}(x), \ldots, \lambda^{k}(x)\right), \text { for all } k,
$$


where $P_{k}$ is the $k^{\text {th }}$ Newton polynomial (see $\left.[9, \S 3]\right)$. Recall $P_{k}$ is a homogeneous polynomial of degree $k$ in $\mathbb{Z}\left[x_{1}, \ldots, x_{k}\right]$ where $\operatorname{deg}\left(x_{i}\right)=i$. We use equation (8) to construct Adams operations on the spaces $K\left(\mathcal{P}_{n}\right)$, for $\mathcal{P}_{n}$ any family of exact categories with exterior power operations. To do this, we utilize the fact that $\left|\mathrm{G} .{ }^{k} \mathcal{P}_{n}\right|$ is an H-space for which multiplication by -1 is defined. Also, there exist external products

$$
\text { G. }{ }^{i} \mathcal{P}_{n} \otimes \mathrm{G} .{ }^{j} \mathcal{P}_{m} \longrightarrow \mathrm{G} \cdot{ }^{i+j} \mathcal{P}_{n+m}
$$

where the symbol $\otimes$ is the external product of multisimplicial sets. We define $\mu_{i, j}$ to be the composite map

$$
\left|\mathrm{G} .{ }^{i} \mathcal{P}_{n}\right| \times\left|\mathrm{G} .{ }^{j} \mathcal{P}_{m}\right| \cong\left|\mathrm{G} .{ }^{i} \mathcal{P}_{n} \otimes \mathrm{G} .{ }^{j} \mathcal{P}_{m}\right| \longrightarrow\left|\mathrm{G} .{ }^{i+j} \mathcal{P}_{n+m}\right|
$$

Thus addition, subtraction and multiplication of maps are defined and we may define

$$
\Psi^{k}: \mid \text { G. } \cdot \mathcal{P}_{n}|\longrightarrow| \mathrm{G}^{k}{ }^{k} \mathcal{P}_{n k} \mid
$$

using the equation (8). To illustrate, the map $\Psi^{2}=2 \lambda^{2}-\left(\lambda^{1}\right)^{2}$ is defined such that

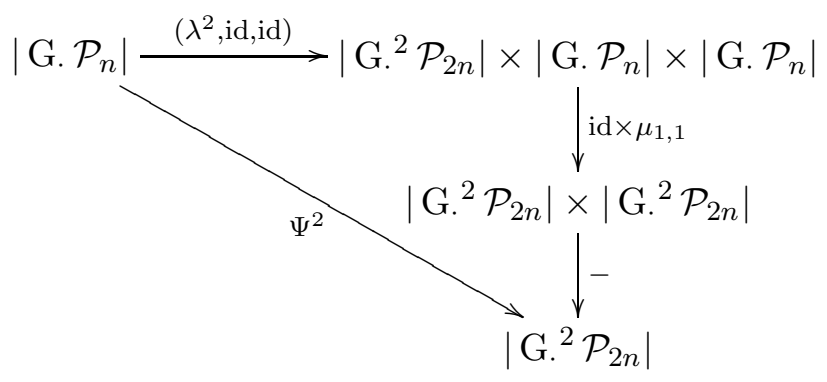


commutes. More generally, if there are more than two terms in a certain Newton polynomial, one must choose, once and for all, the order in which to add these terms together. Also one must choose, once and for all, the order in which to multiply factors in each term. The point of making such choices is to ensure that $\Psi^{k}$ is strictly natural with respect to functors of exact categories with exterior powers. The choices involved will not affect the homotopy class of the resulting map of spaces. The equation (8) holds on the level of homotopy groups as well, but observe that the multiplication involved induces the zero map on all higher homotopy groups. Thus, as maps from $K_{q}\left(\mathcal{P}_{n}\right)$ to $K_{q}\left(\mathcal{P}_{n k}\right)$ for $q>0$, we have the equation $[10, \S 4]$

$$
\Psi^{k}=(-1)^{k+1} k \lambda^{k}
$$

The reader should be warned that the maps $\Psi^{k}$ need not be maps of H-spaces, but they do induce the usual Adams operations associated to the $\lambda$-operations on the level of $K$-groups and are in particular additive on $K_{0}$. 


\section{Adams operations on $K\left(X, \mathbb{P}^{\wedge t}\right)$}

We begin this section by considering the general class of $\mathcal{M}$-schemes formed by taking all the symmetric products of a given quasi-projective scheme $Y$. Eventually, we will consider only the case where $Y$ is the projective line, in which case we exploit the fact that $S^{k} \mathbb{P}^{1} \cong \mathbb{P}^{k}$. For now, assume $Y$ has a distinguished line bundle $\mathcal{L}$. In the case $Y=\mathbb{P}^{1}$, we will take $\mathcal{L}$ to be $\mathcal{O}(-1)$. Let $Y^{n}=\mathrm{S}^{n} Y$ and observe that there is an obvious line bundle on $Y^{n}$ formed by taking the exterior tensor product $\mathcal{L} \otimes \cdots \otimes \mathcal{L}$ of $\mathcal{L}$ with itself $n$ times to give an line bundle on $Y^{\times n}$ and then taking $\Sigma_{n}$-invariants. We write this line bundle as $\mathcal{L}^{n}$. Observe that $\mathcal{L}^{n}=\mathcal{O}_{\mathbb{P}^{n}}(-1)$ in case $Y=\mathbb{P}^{1}$. Tensoring by $\mathcal{L}^{n}$ induces an autoequivalence of $\mathcal{P}\left(X, Y^{n}\right)$. One can easily check that for an object $\mathcal{F}$ of $\mathcal{P}\left(X, Y^{n}\right)$, we have the natural isomorphism

$$
\Lambda^{k}\left(\mathcal{F} \otimes_{Y^{n}} \mathcal{L}^{n}\right) \cong \Lambda^{k}(\mathcal{F}) \otimes_{Y^{n k}} \mathcal{L}^{n k}
$$

After modifying the categories $\mathcal{P}\left(X, Y^{n}\right)$ in a suitable fashion, we can say that the autoequivalences induced by the $\mathcal{L}^{n}$ commute with tensor product and exterior powers. Let us write $\theta(L)$ for the homeomorphisms of $K\left(X, Y^{n}\right)$ induced by tensoring with $L$.

Given an infinite sequence of quasi-projective schemes $Y_{1}, Y_{2}, \ldots$ with distinguished line bundles $\mathcal{L}_{1}, \mathcal{L}_{2}, \ldots$, we can form the infinite 
sequence of maps of spaces

$$
\begin{aligned}
\ldots \longrightarrow & K\left(X \times \Delta^{\bullet}, \mathrm{S}^{n} Y_{1} \times \cdots \times \mathrm{S}^{n} Y_{t+1}\right) \stackrel{\partial}{\longrightarrow} \\
& K\left(X \times \Delta^{\bullet}, \mathrm{S}^{n} Y_{1} \times \cdots \times \mathrm{S}^{n} Y_{t}\right) \longrightarrow \ldots \longrightarrow K\left(X \times \Delta^{\bullet}\right) .
\end{aligned}
$$

The map $\partial$ is given by the difference

$$
p_{t+1}-p_{t+1} \circ \theta\left(\mathcal{L}_{t+1}\right),
$$

where $p_{t+1}$ is the map induced by the evident projection

$$
S^{n} Y_{1} \times \cdots \times S^{n} Y_{t+1} \longrightarrow S^{n} Y_{1} \times \cdots \times S^{n} Y_{t}
$$

of $\mathcal{M}$-schemes. We use the $\mathcal{M}$-scheme structure of $S \cdot Y_{1} \times \cdots \times S \cdot Y_{t}$ to define Adams operations degreewise on the simplicial space

$$
d \mapsto K\left(X \times \Delta^{d}, \mathrm{~S}^{n} Y_{1} \times \cdots \times \mathrm{S}^{n} Y_{t}\right)
$$

which then induce Adams operations on the realized spaces. Observe that in (9) we are using the H-space structure of the $K$-theory spaces involved to realize the difference of the two maps. The Adams operations need not be H-space maps and thus the Adams (and lambda) operations will not commute on the space level with $\partial$, even though they do commute with each constituent map. Nevertheless, the Adams operations will commute with $\partial$ at least on the level of homotopy groups, 
which is all we will need. That is, the diagram

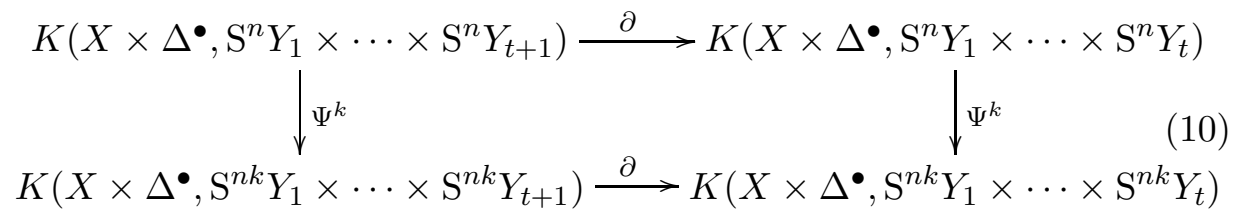

of spaces commutes on homotopy groups. This is a direct consequence of the fact that we have constructed exterior powers to commute strictly with the two functors used to define $\partial$, and the fact that $\Psi^{k}$ is additive on homotopy groups.

Now let us take $Y_{i}=\mathbb{P}^{1}$ and $\mathcal{L}_{i}=\mathcal{O}(-1)$ for all $i$. For all $k$ we have a sequence of maps

$$
\begin{gathered}
\ldots \longrightarrow K\left(X \times \Delta^{\bullet},\left(\mathbb{P}^{k}\right)^{\times t+1}\right) \stackrel{\partial_{k}}{\longrightarrow} K\left(X \times \Delta^{\bullet},\left(\mathbb{P}^{k}\right)^{\times t}\right) \longrightarrow \ldots \\
\longrightarrow K\left(X \times \Delta^{\bullet}\right),
\end{gathered}
$$

and we observe that $\partial$ is equal to the map $\partial_{k}$ defined previously. When $X$ is regular, we are very close to having naturally defined Adams operations on the filtration $W^{t}(X)$ of $K(X) \sim K\left(X \times \Delta^{\bullet}\right)$. Unfortunately, there appears to be a strange degree shift involved in that $\Psi^{k}$ takes $K\left(X \times \Delta^{\bullet}, \mathbb{P}^{1}\right)$ to $K\left(X \times \Delta^{\bullet}, \mathbb{P}^{k}\right)$. The trick is to find a morphism in the category $K_{0}(S c h / \mathbb{Z})$ from $\mathrm{S}^{n} \mathbb{P}^{1}=\mathbb{P}^{n}$ to $\mathbb{P}^{1}$. Consider the closed subscheme $Z_{n} \subset \mathbb{P}^{n} \times \mathbb{P}^{1}$ defined as follows. Let $p: \mathbb{P}^{1} \times \mathbb{P}^{n-1} \longrightarrow \mathbb{P}^{n}$ be the map given by multiplication. This map factors through the projection $\mathbb{P}^{n} \times \mathbb{P}^{1} \longrightarrow \mathbb{P}^{n}$ using the map $\left(p, \pi_{1}\right)$. One can easily show $\left(p, \pi_{1}\right)$ 
is a closed immersion and determines a closed subscheme $Z_{n}$. Observe that the fiber of $Z_{n} \longrightarrow \mathbb{P}^{n}$ over a point of $\mathbb{P}^{n}$, which we think of as an unordered $n$-tuple of points on $\mathbb{P}^{1}$, consists of the $n$ points appearing in this $n$-tuple. Let $S$ and $T$ be homogeneous coordinates of $\mathbb{P}^{1}$. We choose $Z_{0}, \ldots, Z_{n}$ to be homogeneous coordinates on $\mathbb{P}^{n}$ so that under the identification $\mathbb{P}^{n} \cong S^{n} \mathbb{P}^{1}$, an $n$-tuple $\left(\left(x_{1}: y_{1}\right), \ldots,\left(x_{n}: y_{n}\right)\right)$ of points of $\mathbb{P}^{1}$ has for its $i^{\text {th }}$ coordinate, the coefficient of $u^{n-i}$ in the polynomial $\left(x_{1} u+y_{1}\right) \ldots\left(x_{n} u+y_{n}\right)$. The subscheme $Z_{n}$ is therefore defined by the condition that $(S u+T)$ divide $\left(Z_{0} u^{n}+Z_{1} u^{n-1}+\cdots+Z_{n}\right)$, and thus is defined by the single equation

$$
(-1)^{n} T^{n} Z_{n}+(-1)^{n-1} S T^{n-1} Z_{n-1}+\cdots+S^{n} Z_{0}
$$

In particular, the map $Z_{n} \longrightarrow \mathbb{P}^{n}$ is finite and flat of degree $n$, so that the coherent sheaf $\mathcal{O}_{Z_{n}}$ belongs to $\mathcal{P}\left(\mathbb{P}^{n}, \mathbb{P}^{1}\right)$. For technical reasons that will arise later, it turns out that the element we want in $K_{0}\left(\mathbb{P}^{n}, \mathbb{P}^{1}\right)$ is the class of $\mathcal{O}_{Z_{n}} \otimes_{\mathbb{P}^{n} \times \mathbb{P}^{1}} \mathcal{O}(0,-1)$. Let us call this element $\gamma_{n}$.

LEMMA 6.1. The diagram in $K_{0}(S c h / \mathbb{Z})$

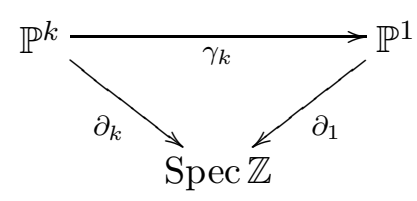

commutes.

Proof. Intuitively, the intersection of $Z_{k}$ with $\mathbb{P}^{k} \times\{P\}$ is a hyperplane of $\mathbb{P}^{k}$, for the general rational point $P$ of $\mathbb{P}^{1}$. More formally, we 
need to compute the element of $K_{0}\left(\mathbb{P}^{k}\right)$ given by $\mu_{*} \pi_{2}^{*}(1-[\mathcal{O}(-1)])$, where $\pi_{2}$ is the projection $\mathbb{P}^{k-1} \times \mathbb{P}^{1} \longrightarrow \mathbb{P}^{1}$ and $\mu$ is the multiplication map $\mathbb{P}^{k-1} \times \mathbb{P}^{1} \longrightarrow \mathbb{P}^{k}$. In light of equation (12), this amounts to computing the image of $\left[\mathcal{O}_{Z_{k}}(0,-1)\right] \cdot(1-[\mathcal{O}(0,-1)]=([\mathcal{O}(0,-1)]-$ $[\mathcal{O}(-1,-k-1)]) \cdot\left(1-[\mathcal{O}(0,-1)]\right.$ under the map $K_{0}\left(\mathbb{P}^{k} \times \mathbb{P}^{1}\right) \longrightarrow K_{0}\left(\mathbb{P}^{k}\right)$ induced by projection. A straightforward calculation finishes the proof.

Currently we have constructed our Adams operations to give maps

$$
\Psi^{k}: K\left(X, \mathbb{P}^{1} \times \cdots \times \mathbb{P}^{1}\right) \longrightarrow K\left(X, \mathbb{P}^{k} \times \cdots \times \mathbb{P}^{k}\right)
$$

which involve an apparent weight shift. We compose this map with the maps obtained by applying $\gamma_{k}$ to each of the $t$ factors of $\mathbb{P}^{k}$. This yields a map $K\left(X,\left(\mathbb{P}^{1}\right)^{\times t}\right) \longrightarrow K\left(X,\left(\mathbb{P}^{1}\right)^{\times t}\right)$. We wish to define the Adams operation $\Psi^{k}$ on the homotopy groups of $W^{t}(X)$ by using this composition of maps. We need to be a little careful here because the Adams operations are not maps of spectra. To handle this, we want to now identify $K\left(X, \mathbb{P}^{\wedge t}\right)$ with a an iterated homotopy fiber of a cube of spaces. Let $Y_{i}$ be either the projective line or a point. Observe that $\Psi^{k}$ acts on the graded simplicial space

$$
d, k \mapsto K\left(X \times \Delta^{d}, S^{k} Y_{1} \times \cdots \times S^{k} Y_{t}\right)
$$


in a fashion compatible with the simplicial structure. Further, the action of $\Psi^{k}$ is compatible with both the inclusion $\{1\} \hookrightarrow \mathbb{P}^{1}$ and the structure map $\mathbb{P}^{1} \longrightarrow\{1\}$.

If allow the $Y_{i}$ to vary, we have that $\Psi^{k}$ acts on (13) viewed as a graded split cube of simplicial spaces. Let take geometric realizations of the spaces in (13) using the simplicial directions. This results in a graded split cube of spaces. Now take iterated homotopy fibers using the split surjections $\mathbb{P}^{k}=S^{k} \mathbb{P}^{1} \longrightarrow S^{k}\{1\}=$ Spec $\mathbb{Z}$. This results in a collection of spaces indexed by $k$. Let us write this as $K\left(X \times \Delta^{\bullet}, S^{n} \mathbb{P}^{\wedge t}\right)$, where we defined $S^{n} \mathbb{P}^{\wedge t}$ to be $S^{n} \mathbb{P}^{\wedge 1} \otimes \cdots \otimes S^{n} \mathbb{P}^{\wedge 1}$ and we interpret $S^{n} \mathbb{P}^{\wedge 1}$ to be the split 1-cube $S^{n}$ Spec $\mathbb{Z} \hookrightarrow S^{n} \mathbb{P}^{1}$. Observe that there is an induced action of $\Psi^{k}$ on this space. To obtain an action of $\Psi^{k}$ on the space $K\left(X \times \Delta^{\bullet}, \mathbb{P}^{\wedge t}\right)$ viewed as an iterated homotopy fiber, we employ the following lemma.

LEMMA 6.2. In the category $K_{0}(S c h / \mathbb{Z})$, the diagrams

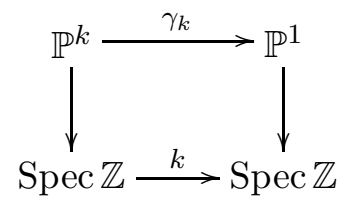

and

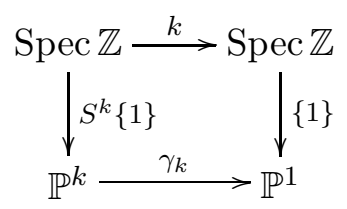

both commute. 
Proof. The composition $\mathbb{P}^{k} \longrightarrow \mathbb{P}^{1} \longrightarrow$ Spec $\mathbb{Z}$ is given by the image under $K_{0}\left(\mathbb{P}^{k} \times \mathbb{P}^{1}\right) \longrightarrow K_{0}\left(\mathbb{P}^{k}\right)$ of $\gamma_{k}$ viewed as an element of $K_{0}\left(\mathbb{P}^{k} \times\right.$ $\left.\mathbb{P}^{1}\right)$. A straightforward calculation shows that this image is $k[\mathcal{O}]$. (Here is where we need to use $\gamma_{k}$ instead of the seemingly more natural map induced by $\left[\mathcal{O}_{Z_{k}}\right]$.)

For the second diagram, using formula (12), we observe that the pullback of $\mathcal{O}_{Z_{k}}(0,-1)$ under the closed immersion

$$
\mathbb{P}^{1} \cong\{1\} \times \mathbb{P}^{1} \longrightarrow \mathbb{P}^{k} \times \mathbb{P}^{1}
$$

is the structure sheaf of the closed subscheme defined by $(S-T)^{k}$ twisted by $\mathcal{O}(-1)$. The twisting has no effect since the sheaf is supported on a point and we see that the second diagram commutes.

We can therefore define a map in $K_{0}(S c h / \mathbb{Z})$ of split cubes from $S^{k} \mathbb{P}^{\wedge t}$ to $\mathbb{P}^{\wedge t}$, where for each $I$, the map $S^{k}\left(\mathbb{P}_{I}^{\wedge t}\right) \longrightarrow \mathbb{P}_{I}^{\wedge t}$ is $k^{|I|-t}$ times the map given by applying $\gamma_{k}$ to each factor of $\mathbb{P}^{k}$ that appears. The action of $\Psi^{k}$ on $K\left(X \times \Delta^{\bullet}, \mathbb{P}^{\wedge t}\right)$ is therefore given as the composition

$$
K\left(X \times \Delta^{\bullet}, \mathbb{P}^{\wedge t}\right) \longrightarrow K\left(X \times \Delta^{\bullet}, S^{k} \mathbb{P}^{\wedge t}\right) \longrightarrow K\left(X \times \Delta^{\bullet}, \mathbb{P}^{\wedge t}\right) .
$$

The action of $\Psi^{k}$ on the space $K\left(X \times \Delta^{\bullet}, \mathbb{P}^{\wedge t}\right)$ is thus realized as a map of simplicial split cubes of spaces. The factors of $k^{|I|-t}$ introduced can be justified in light of the Riemann-Roch theorem, but in any case they are not a cause for alarm since the map $K\left(X \times \Delta^{\bullet},\left(\mathbb{P}^{1}\right)^{\times t+1}\right) \longrightarrow$ 
$K\left(X \times \Delta^{\bullet},\left(\mathbb{P}^{1}\right)^{\times t}\right)$ annihilates all but the summand $W^{t+1}(X)$ on the level of homotopy groups.

THEOREM 6.3. The Adams operations are compatible with the filtration

$$
\cdots \longrightarrow K\left(X \times \Delta^{\bullet}, \mathbb{P}^{\wedge t+1}\right) \stackrel{\partial_{1}}{\longrightarrow} K\left(X \times \Delta^{\bullet}, \mathbb{P}^{\wedge t}\right) \longrightarrow \ldots \longrightarrow K(X) .
$$

on the level of homotopy groups.

Proof. It suffices to show that Adams operations are compatible with the map $K\left(X \times \Delta^{\bullet},\left(\mathbb{P}^{1}\right)^{\times t+1}\right) \longrightarrow K\left(X \times \Delta^{\bullet},\left(\mathbb{P}^{1}\right)^{\times t}\right)$ on the level of homotopy groups since the map $\partial_{1}$ annihilates all but the summand $\pi_{n} K\left(X \times \Delta^{\bullet}, \mathbb{P}^{\wedge t}\right)$. We already know that the diagram

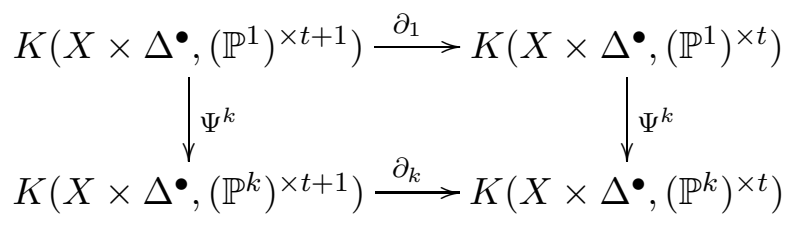

commutes on the level of homotopy groups. Now apply Lemma 6.1.

We now relate the Adams operations on $K\left(X, \mathbb{P}^{\wedge t}\right)$ with operations on $K\left(X, \mathbb{G}_{\mathrm{m}}^{\wedge t}\right)$. This latter spectrum is the realization of the cube of spectra obtained by applying $K(X,-)$ degreewise to the cube of schemes $\mathbb{G}_{\mathrm{m}}^{\wedge t}$. The cube $\mathbb{G}_{\mathrm{m}}^{\wedge t}$ is defined by first declaring $\mathbb{G}_{\mathrm{m}}^{\wedge 1}$ to be the 1-cube

$$
\{1\} \longrightarrow \mathbb{P}^{1}-\{0, \infty\}
$$


and then letting $\mathbb{G}_{\mathrm{m}}^{\wedge t}=\mathbb{G}_{\mathrm{m}}^{\wedge 1} \nabla \cdots \otimes \mathbb{G}_{\mathrm{m}}^{\wedge 1}$. The $t$-fold delooping of the spectrum $K\left(\operatorname{Spec} R \times \Delta^{\bullet}, \mathbb{G}_{\mathrm{m}}^{\wedge t}\right)$ is $G W^{t}(R)$, the $t^{\text {th }}$ stage of Grayson's filtration. In [19], we establish a map

$$
K\left(X \times \Delta^{\bullet}, \mathbb{G}_{\mathrm{m}}^{\wedge t}\right) \longrightarrow \Omega^{t} K\left(X \times \Delta^{\bullet}, \mathbb{P}^{\wedge t}\right)
$$

which is a weak equivalence when $X$ is the spectrum of the localization at a prime of a smooth algebra of finite type over a perfect field. Observe that $\mathbb{G}_{\mathrm{m}}^{\wedge 1}$ is split by the structure map $\mathbb{G}_{\mathrm{m}}^{1} \longrightarrow$ Spec $k$. We regard $\mathbb{G}_{\mathrm{m}}^{\wedge 1}$ as a split 1-cube using this structure map and furthermore we use this map to regard $S^{k} \mathbb{G}_{\mathrm{m}}^{\wedge 1} \otimes \cdots \otimes S^{k} \mathbb{G}_{\mathrm{m}}^{\wedge 1}$ as a split cubes We will also have occasion to use the open subschemes $\mathbb{A}^{1} \cong \mathbb{P}^{1}-\{0\}$ and $\mathbb{A}^{1} \cong \mathbb{P}^{1}-\{\infty\}$ in place of $\mathbb{P}^{1}$. Observe that the $n^{\text {th }}$ symmetric power of $\mathbb{A}^{1}$ is isomorphic to $\mathbb{A}^{n}$. We regard $S^{n} \mathbb{A}^{\wedge 1} \otimes \cdots \otimes S^{n} \mathbb{A}^{\wedge 1}$ as a split cube of schemes using the inclusion $\{1\} \longrightarrow \mathbb{A}^{1}$ and the structure morphism.

The category $\mathcal{P}\left(X, \mathbb{G}_{\mathrm{m}}^{i}\right)$ is equivalent to the category consisting of locally free sheaves on $X$ equipped with an $i$-tuple of pairwise commuting automorphisms. Using exterior powers of vector bundles and the induced exterior powers of automorphisms, we have an obvious choice for exterior power operations on the category $\mathcal{P}\left(X, \mathbb{G}_{\mathrm{m}}^{i}\right)$ which does not rely on the complicated bivariant definition described earlier in this paper. Alternatively, these evident exterior power functors arise 
using the bivariant construction introduced earlier and the fact that $\mathbb{G}_{\mathrm{m}}^{1}$ is a monoid.

THEOREM 6.4. The action of the Adams operations is compatible with the natural map

$$
K\left(X \times \Delta^{\bullet}, \mathbb{G}_{m}^{\wedge k}\right) \longrightarrow \Omega^{k} K\left(X \times \Delta^{\bullet}, \mathbb{P}^{\wedge k}\right)
$$

on the level of homotopy groups.

Remark 6.5. The Adams operations on the left hand side are induced by exterior powers of automorphisms. The definition of the map (14) will be recalled in the course of the proof.

Proof. For the moment, we define the action of the Adams operations on $K\left(X \times \Delta^{\bullet}, S \cdot \mathbb{G}_{\mathrm{m}}^{1} \times \cdots \times S \cdot \mathbb{G}_{\mathrm{m}}^{1}\right)$ as defined in Section 5. As for the case with copies of $\mathbb{P}^{1}$, we induce an action of $\Psi^{k}$ on the space

$$
K\left(X \times \Delta^{\bullet}, S \cdot \mathbb{G}_{\mathrm{m}}^{\wedge 1} \times \cdots \times S \cdot \mathbb{G}_{\mathrm{m}}^{\wedge 1}\right),
$$

which is defined as the iterated homotopy fiber of the cube involving the split surjections $S^{n} \mathbb{G}_{\mathrm{m}}^{1} \longrightarrow S^{n}\{1\} \cong$ Spec $\mathbb{Z}$. We will eventually relate there operations to those induced by exterior power of automorphisms. Our first goal is to show the Adams operations on the space (15) are compatible with the Adams operations on the space

$$
K\left(X^{\bullet}, S \cdot \mathbb{P}^{\wedge 1} \times \cdots \times S \cdot \mathbb{P}^{\wedge 1}\right) .
$$


Fix coordinates on $\mathbb{P}^{1}$ and consider the usual two copies of $\mathbb{A}^{1}$ in $\mathbb{P}^{1}$ and their intersection $\mathbb{G}_{\mathrm{m}}^{1}$. Taking symmetric powers, we get a square of $\mathcal{M}$-schemes

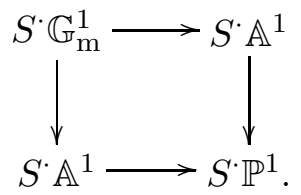

Let this square be written as $C^{1}$. Applying the functor $K\left(X \times \Delta^{\bullet},-\right)$ to $C^{1}$ results in a square of spaces and the operations $\lambda^{k}$ act on this square (provided we are careful in choosing the models for the $K$-theory spaces).

There is a map from $\operatorname{Spec} \mathbb{Z}$ to each of the four $\mathcal{M}$-schemes appearing in (16) induced by inclusion at the point with homogeneous coordinates $[1: 1]$, and these maps are split by the structure morphisms. Let $C^{\wedge 1}$ denote the square of split 1-cubes of $\mathcal{M}$-schemes with corners $S \cdot \mathbb{G}_{\mathrm{m}}^{\wedge 1}, S \cdot \mathbb{A}^{\wedge 1}, S \cdot \mathbb{A}^{\wedge 1}$, and $S \cdot \mathbb{P}^{\wedge 1}$, where given a cube of schemes $C$, we interpret $S \cdot C$ to be the cube of graded schemes obtained by applying $S$ degreewise to the cube. So $C^{\wedge 1}$ is the three-dimensional cube of 
$\mathcal{M}$-schemes

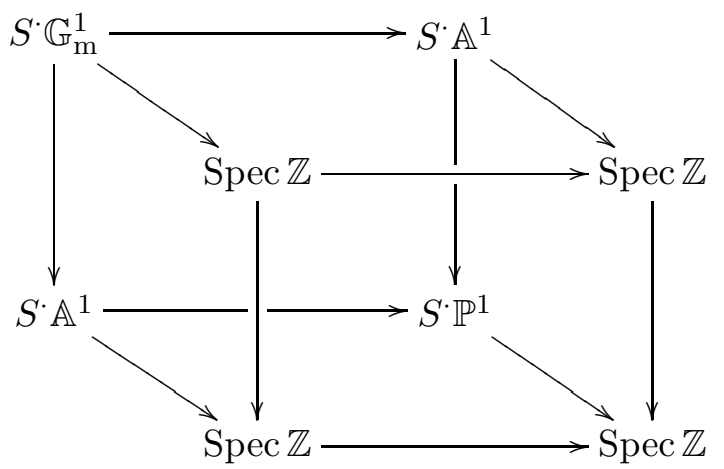

Moreover, the diagonal arrows admit a compatible set of splittings.

Given an $t$-cube of $\mathcal{M}$-schemes $D$, define a square of $(t+1)$-cubes of $\mathcal{M}$-schemes $D \times C^{\wedge 1}$ with corners $D \otimes S \cdot \mathbb{G}_{\mathrm{m}}^{\wedge 1}, D \otimes S \cdot \mathbb{A}^{\wedge 1}, D \otimes S \cdot \mathbb{A}^{\wedge 1}$, and $D \otimes S \cdot \mathbb{P}^{\wedge 1}$. Now observe that $K\left(X \times \Delta^{\bullet}, D \otimes S \cdot \mathbb{A}^{\wedge 1}\right)$ is a contractible space, since the functor $K\left(X \times \Delta^{\bullet},-\right)$ is homotopy invariant and $S^{d} \mathbb{A}^{1} \cong \mathbb{A}^{d}$. The square $K\left(X \times \Delta^{\bullet}, D \times C^{\wedge 1}\right)$, which one obtains by applying the functor $K\left(X \times \Delta^{\bullet},-\right)$ to each of the four corners of $D \times C^{\wedge 1}$, therefore induces a map

$$
K\left(X \times \Delta^{\bullet}, D \times S \cdot \mathbb{G}_{\mathrm{m}}^{\wedge 1}\right) \longrightarrow \Omega K\left(X \times \Delta^{\bullet}, D \times S \cdot \mathbb{P}^{\wedge 1}\right)
$$

The model for the space

$$
\Omega K\left(X \times \Delta^{\bullet}, D \times S^{n} \mathbb{P}^{\wedge 1}\right)
$$


is the homotopy pullback of

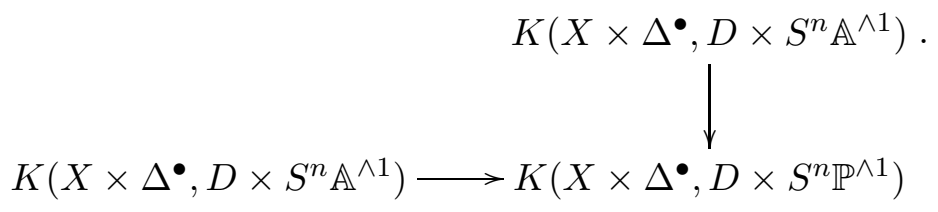

Further, for suitable models of the spaces appearing in $K\left(X \times \Delta^{\bullet}, D \times\right.$ $C^{\wedge 1}$ ), the $\lambda$-operations act on this square of spaces, and thus we have a commutative square

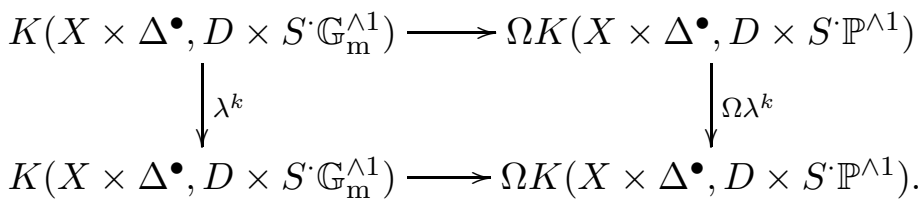

By induction, it follows that there is a natural map

$K\left(X \times \Delta^{\bullet}, S^{\cdot} \mathbb{G}_{\mathrm{m}}^{\wedge 1} \otimes \cdots \otimes S^{\cdot} \mathbb{G}_{\mathrm{m}}^{\wedge 1}\right) \longrightarrow \Omega^{t} K\left(X \times \Delta^{\bullet}, S \cdot \mathbb{P}^{\wedge 1} \otimes \cdots \otimes S^{\wedge} \mathbb{P}^{\wedge 1}\right)$

compatible with the $\lambda$-operations. This is the map that is shown to be a weak equivalence locally on a smooth scheme over a prefect field in [19]. We have therefore achieved our first goal.

We need to understand the map $\gamma_{k}$ in $K_{0}(S c h / \mathbb{Z})$ from $S^{k} \mathbb{P}^{1}=\mathbb{P}^{k}$ to $\mathbb{P}^{1}$ when restricted to the open subset $\mathrm{S}^{k} \mathbb{G}_{\mathrm{m}}^{1}$. Let us call this restriction $\beta_{k}$ and observe that $\beta_{k}$ factors through $\mathbb{G}_{\mathrm{m}}^{1}$, since the intersection of $\mathrm{S}^{k} \mathbb{G}_{\mathrm{m}}^{1} \times \mathbb{P}^{1}$ and the support of the coherent sheaf defining $\gamma_{k}$ is contained in $\mathrm{S}^{k} \mathbb{G}_{\mathrm{m}}^{1} \times \mathbb{G}_{\mathrm{m}}^{1}$. We also have that $\mathrm{S}^{k} \mathbb{G}_{\mathrm{m}}^{1} \cong \mathbb{A}^{k-1} \times \mathbb{G}_{\mathrm{m}}^{1}$. Using equation 
(12) and the fact that $\left.\mathcal{O}(0,-1)\right|_{S^{k} \mathbb{G}_{\mathrm{m}}^{1} \times \mathbb{G}_{\mathrm{m}}^{1}}$ is the trivial line bundle, we see that the map $\beta_{k}$ is represented by the bimodule

$$
\mathbb{Z}\left[x_{1}, \ldots, x_{k}, x_{k}^{-1}, y, y^{-1}\right] /<y^{k}-x_{1} y^{k-1}+\cdots+(-1)^{k} x_{k}>
$$

By considering the defining relation

$$
s\left(y^{k}-x_{1} y^{k-1}+\cdots+(-1)^{k} x_{k}\right)+(1-s)\left(y-x_{k}\right)(y-1)^{k-1},
$$

with $s$ a variable, we can deform $\beta_{k}$ to the map given by the bimodule

$$
\mathbb{Z}\left[x_{1}, \ldots, x_{k}, x_{k}^{-1}, y, y^{-1}\right] /\left(y-x_{k}\right)(y-1)^{k-1} .
$$

The class of this bimodule in $K_{0}\left(\mathbb{G}_{\mathrm{m}}^{1} \times \mathbb{A}^{k-1}, \mathbb{G}_{\mathrm{m}}^{1}\right)$ is equal to the class of the natural projection of $\mathbb{G}_{\mathrm{m}}^{1} \times \mathbb{A}^{k-1}$ to $\mathbb{G}_{\mathrm{m}}^{1}$ plus $k-1$ times the constant map from $\mathbb{G}_{\mathrm{m}}^{1} \times \mathbb{A}^{k-1}$ to a point. Further, the projection $\mathbb{G}_{\mathrm{m}}^{1} \times \mathbb{A}^{k-1} \longrightarrow$ $\mathbb{G}_{\mathrm{m}}^{1}$ corresponds to the map $\mathrm{S}^{k} \mathbb{G}_{\mathrm{m}}^{1} \longrightarrow \mathbb{G}_{\mathrm{m}}^{1}$ induced by the monoid structure on $\mathbb{G}_{\mathrm{m}}^{1}$. The constant map from $S^{k} \mathbb{G}_{\mathrm{m}}^{1}$ to $\mathbb{G}_{\mathrm{m}}^{1}$ which factors though $\{1\}$ induces the zero map after taking homotopy groups of the $\operatorname{map} K\left(X, S^{k}\left(\mathbb{G}_{\mathrm{m}}^{\wedge 1}\right)\right) \longrightarrow K\left(X, \mathbb{G}_{\mathrm{m}}^{\wedge 1}\right)$.

In summary, the diagram

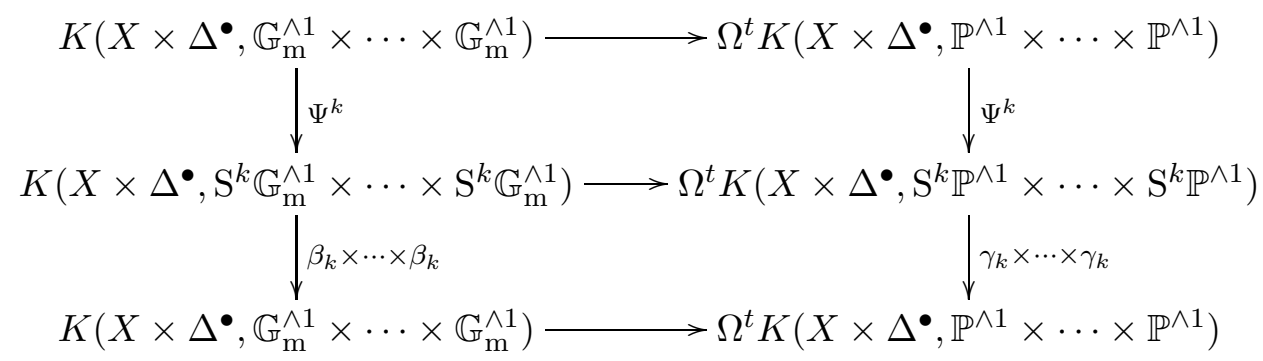


commutes up to homotopy. Further, the lower left vertical arrow is homotopic to the sum of the map induced by the monoid structure on $\mathbb{G}_{\mathrm{m}}^{1}$ and $k-1$ times the map induced by the constant map $S^{k} \mathbb{G}_{\mathrm{m}}^{1} \longrightarrow$ $\{1\}$. On homotopy groups, the lower left vertical arrow is therefore given by the monoid structure on $\mathbb{G}_{\mathrm{m}}^{1}$, and so the composition of the vertical arrows on the left gives the Adams operations on $K$-groups with automorphisms, and the theorem is proven.

Using the Theorem, we achieve a result that seems very difficult to show without considering the filtration $W^{t}(X)$.

COROLLARY 6.6. The map $K\left(X \times \Delta^{\bullet}, \mathbb{G}_{m}^{\wedge t+1}\right) \longrightarrow \Omega K\left(X \times \Delta^{\bullet}, \mathbb{G}_{m}^{\wedge t}\right)$ used in Grayson's filtration of $K(X)$ is compatible on the level of homotopy groups with the Adams operations provided $X$ is the spectrum of the localization at a prime of a smooth algebra of finite type over a perfect field.

\section{The weight filtration $W^{t}$}

The goal of this section to give a partial answer to the question of whether Grayson's filtration on $K(X)$ is truly a weight filtration in

the sense that the $t^{\text {th }}$ stage of the filtration gives the weight $\geq t$ part 
of $K$-theory. Actually, we will work exclusively with the filtration on $K(R)$ whose $t^{\text {th }}$ term is $W^{t}(X)=K\left(X \times \Delta^{\bullet}, \mathbb{P}^{\wedge t}\right)$. We establish two different facts concerning the weights of this filtration. First, we show that for $k$ an arbitrary field, the weight $t$ part of $\pi_{q} W^{t}(k)$ is isomorphic to the weight $t$ part of $K_{q}(k)$. Oddly, it is difficult to determine if the isomorphism constructed is in fact induced by the map $W^{t}(\operatorname{Spec} k) \longrightarrow$ $K(\operatorname{Spec} k)$. Second, using none of the techniques introduced in this paper so far, we show that the filtration on $K_{q}(X)$ whose $t^{\text {th }}$ stage is defined by $\operatorname{im}\left(\pi_{q} W^{t}(X) \longrightarrow K_{q}(X)\right)$ gives, after tensoring with the rationals, the gamma filtration, when $X$ is a smooth variety over a field $k$ for which resolution of singularities holds.

Let $R$ be a ring and make the convention $W^{t}(R)=W^{t}(\operatorname{Spec} R)$. So far we have constructed Adams operations on $W^{t}(R)$ compatible with the maps of the filtration at least on the level of homotopy groups. But we know very little about these operations, not even if rationally they decompose the homotopy groups into eigenspaces. To tackle this problem, let us establish a convenient category to work in. To begin with, we consider the abelian category of all $\mathbb{Q}$-vector spaces $V$ that are equipped with operations $\Psi^{k}$, for $k \geq 0$, and satisfy the property that all the eigenvalues of $\Psi^{k}$ (over $\overline{\mathbb{Q}}$ ) lie in the set $\left\{1, k, k^{2}, \ldots\right\}$ and such that any element of $V$ is a sum of generalized eigenvectors - i.e. vectors killed by $\left(\Psi^{k}-k^{i}\right)^{N}$ for some $i$ and $N$. Equivalently, we could stipulate 
that for each $k$, every element of $V$ is killed by a finite product of (not necessarily distinct) endomorphisms of the form $\left(\Psi^{k}-k^{i}\right)$. Let us call this category $\mathcal{B}$. Fixing a $k$, an object $V$ of $\mathcal{B}$ can be decomposed into generalized eigenspaces

$$
V^{(i)}:=\left\{v \in V \mid\left(\Psi^{k}-k^{i}\right)^{N}(v)=0 \text { for } N \gg 0\right\}
$$

since $V^{(i)} \cap V^{(j)}=0$ for $i \neq j$. We want to consider just those objects $V$ of $\mathcal{B}$ for which the subspace $V^{(i)}$ independent of $k$, for all $i$. Let us call the full subcategory of $\mathcal{B}$ consisting of such objects the category of $\mathbb{Q}$ vector spaces with Adams operations or $A \mathbb{Q}$. We will call the subspace $V^{(i)}$ the weight $i$ part of $V$. We say $V$ is pure if $V^{(i)}$ consists entirely of eigenvectors. In this case, we call the the collection of integers $i$ such that $V^{(i)}$ is nonzero the weights of $V$, and $V$ is pure of weight $i$ if $i$ is the only weight of $V$.

LEMMA 7.1. The functor $V \mapsto V^{(i)}$ is an exact functor on the category $A \mathbb{Q}$. The category $A \mathbb{Q}$ is a Serre subcategory of the category of all $\mathbb{Q}$-vector spaces equipped with endomorphisms $\Psi^{k}$ (but not necessarily satisfying the condition on the eigenvalues).

Proof. Observe that a $\mathbb{Q}$-vector space $V$ equipped with endomorphisms $\Psi^{k}$ belongs to $\mathcal{B}$ if and only if when regarded as a $\mathbb{Q}[x]$-module with $x$ acting as $\Psi^{k}$, every finitely generated submodule is annihilated by a product of linear polynomials of the form $x-k^{i}$. This shows that 
both claims of the lemma hold for the category $\mathcal{B}$. Certainly, the first claim holds for $A \mathbb{Q}$ as well. To establish the second claim for $A \mathbb{Q}$, it suffices to show $A \mathbb{Q}$ is a Serre subcategory of $\mathcal{B}$, which is straightforward.

If $k$ is an algebraically closed field, the free abelian group on the $k$ rational points of monoid $S \cdot \mathbb{P}^{1}$ forms a polynomial ring with generators elements of the set $\mathbb{P}^{1}(k)$. We define lambda operations on a polynomial ring over $\mathbb{Z}$ by declaring that monomials have rank 1 - i.e. $\lambda^{k}(m)=0$ for $k>1, m$ a monomial. This is in fact a special lambda ring and the induced Adams operation $\Psi^{k}$ is the ring map which raises all monomials to the $k^{\text {th }}$ power. For in general, given a set $S$, the polynomial ring $A=\mathbb{Z}[S]$ embeds in the $\lambda$-ring $1+A[[t]]^{+}$by sending $s \in S$ to $1+s t$ and this embedding determines the $\lambda$-ring structure of A. See [1] for more details.

LEMMA 7.2. Let $k$ be a field, $E$ the field of fractions of $\mathbb{A}_{k}^{n}$, for some n, and $\bar{E}$ an algebraic closure of $E$. Then for any quasi-projective scheme $Y$, the $\operatorname{map} K_{q}\left(\mathbb{A}_{k}^{n}, Y\right)_{\mathbb{Q}} \longrightarrow K_{q}(\bar{E}, Y)_{\mathbb{Q}}$ is an injection. Moreover, we have

$$
\begin{aligned}
K_{q}\left(\bar{E}, S^{\cdot} \mathbb{P}^{1} \times \cdots \times S^{\cdot} \mathbb{P}^{1}\right)_{\mathbb{Q}} & \cong K_{0}\left(\bar{E}, S \cdot \mathbb{P}^{1} \times \cdots \times S^{\cdot} \mathbb{P}^{1}\right) \otimes K_{q}(\bar{E}) \\
& \cong \mathbb{Z}\left[\mathbb{P}^{1}(\bar{E})\right] \otimes \cdots \otimes \mathbb{Z}\left[\mathbb{P}^{1}(\bar{E})\right] \otimes K_{q}(\bar{E}),
\end{aligned}
$$


and the Adams operations are compatible with these isomorphisms. In particular,

$$
K_{q}\left(\mathbb{A}_{k}^{n}, S \cdot \mathbb{P}^{1} \times \cdots \times S \cdot \mathbb{P}^{1}\right)_{\mathbb{Q}}
$$

is a special lambda ring.

Proof. Let $X=\mathbb{A}_{k}^{n}$. For any $k$-scheme $Y$, let $\mathcal{M}(X, Y)$ denote the category of coherent sheaves on $X \times_{k} Y$ whose supports map finitely to $X$. Recall that $K^{\prime}(X, Y)$ denotes the associated $K$-theory space. Now let $Y$ be a product of projective spaces. Then we know $K(X, Y) \sim K^{\prime}(X, Y)$ under the evident map. Further, if $\mathcal{M}^{1}(X, Y)$ denotes the Serre subcategory of $\mathcal{M}(X, Y)$ consisting of sheaves whose support maps (finitely) to a proper closed subset of $X$, then using a linear version of the Gersten conjecture [19, Theorem 3.8], the maps on $K$-groups induced by the inclusion $\mathcal{M}^{1}(X, Y) \longrightarrow \mathcal{M}(X, Y)$ are zero. Further, observe that the quotient category $\mathcal{M}(X, Y) / \mathcal{M}^{1}(X, Y)$ is equivalent to the full subcategory of $\mathcal{M}(\operatorname{Spec} E, Y)$ consisting of sheaves supported on some $Z \subset$ Spec $E \times Y$ such that the closure of $Z$ in $X \times Y$ is finite over $X$. Let us write this category as $\mathcal{M}^{X}(\operatorname{Spec} E, Y)$.

More generally, if $R$ is a normal integral domain with field of fractions $L$, we let $K_{q}^{R}(L, Y)$ denote the $K$-group associated to the full subcategory of $\mathcal{M}(\operatorname{Spec} L, Y)$ consisting of sheaves supported on a $Z$ whose closure in $\operatorname{Spec} R \times Y$ is finite over $\operatorname{Spec} R$. Now let $L \subset L^{\prime}$ be 
a finite field extension, and let $R^{\prime}$ be the integral closure of $R$ in $L^{\prime}$. Then the induced map

$$
K_{q}^{R}(L, Y)_{\mathbb{Q}} \longrightarrow K_{q}^{R^{\prime}}\left(L^{\prime}, Y\right)_{\mathbb{Q}}
$$

is an injection, since composition of this map with the transfer map defined by restricting scalars induces multiplication by the degree of the field extension. For this argument to make sense, one needs to check that restriction of scalars does indeed induce a functor from $\mathcal{M}^{R^{\prime}}\left(L^{\prime}, Y\right)$ to $\mathcal{M}^{R}(L, Y)$, but this is an easy consequence of the fact that $R^{\prime} \cap L=$ $R$.

Taking a limit over all finite field extensions of $L$, we get an injection

$$
K_{q}^{R}(L, Y)_{\mathbb{Q}} \longrightarrow K_{q}^{\bar{R}}(\bar{L}, Y)_{\mathbb{Q}}
$$

where $\bar{R}$ denotes the absolute integral closure of $R$. Observe that $\mathcal{M}^{\bar{R}}(\bar{L}, Y)$ is an abelian category and every object of it admits a finite filtration whose quotients are objects of the form $\mathcal{O}_{y}$, for a closed (and hence $\bar{L}$ rational) point of $Y_{\bar{L}}$. In fact, if we embed $Y$ in some projective space, the quotients that appear correspond to those points of $Y_{\bar{L}}$ that have coordinates in $\bar{R}$. Let $Y^{\bar{R}}(\bar{L})$ denote this collection of closed points of $Y_{\bar{L}}$. Consider the abelian category

$$
\mathcal{A}:=\bigoplus_{Y^{\bar{R}}(\bar{L})} \mathcal{M}(\bar{L})
$$


We may identify $\mathcal{A}$ with the full subcategory of $\mathcal{M}^{\bar{R}}(\bar{L}, Y)$ consisting of those coherent sheaves which are a finite direct sum of sheaves of the form $\mathcal{O}_{y}$ for $y \in Y^{\bar{R}}(\bar{L})$, since there are no nonzero homomorphisms from $\mathcal{O}_{y}$ to $\mathcal{O}_{y^{\prime}}$ for $y \neq y^{\prime}$. Observe that $\mathcal{A}$ is closed under taking subobjects, quotient objects, and finite products in $\mathcal{M}^{\bar{R}}(\bar{L}, Y)$. Since every object of $\mathcal{M}^{\bar{R}}(\bar{L}, Y)$ admits a finite filtration whose quotients lie in $\mathcal{A}$, Quillen's Devissage Theorem [13, Theorem 4] shows that

$$
K_{q}(\mathcal{A}) \cong K_{q}^{\bar{R}}(\bar{L}, Y)
$$

In particular, the map $K_{q}^{\bar{R}}(\bar{L}, Y) \longrightarrow K_{q}(\bar{L}, Y)$ is an injection, since the left hand side (respectively, the right hand side) is isomorphic to a direct sum of copies of $K_{q}(\bar{L})$ indexed by the set $Y^{\bar{R}}(\bar{L})$ (respectively, $Y(\bar{L})$ ). Combining these observations, we arrive at the conclusion that

$$
K_{q}\left(\Delta^{d}, Y\right)_{\mathbb{Q}} \longrightarrow K_{q}(\bar{E}, Y)_{\mathbb{Q}}
$$

is an injection.

Let $F$ be any algebraically closed field and consider the lambda operations

$$
\lambda^{k}: K_{0}\left(F, \mathbb{P}^{n} \times \cdots \times \mathbb{P}^{n}\right) \longrightarrow K_{0}\left(F, \mathbb{P}^{n k} \times \cdots \times \mathbb{P}^{n k}\right)
$$

The group $K_{0}\left(F, \mathbb{P}^{n} \times \cdots \times \mathbb{P}^{n}\right)$ is free abelian on the set of closed points $\mathbb{P}^{n}(F)^{\times t}$. Identify $F$-rational points of $\mathbb{P}^{n}(F)$ with an unordered $n$-tuple of $F$-rational points of $\mathbb{P}^{1}(F)$, and write such a point as $P_{1} \bullet$ 
$\cdots \bullet P_{n}$. It is immediate from our construction of exterior powers on $\mathcal{P}\left(R, S \cdot \mathbb{P}^{1} \times \cdots \times S \cdot \mathbb{P}^{1}\right)$ that the $k^{\text {th }}$ exterior power of $F$-vector space $V$ supported on the $F$-rational point

$$
y=\left(P_{1,1} \bullet \cdots \bullet P_{1, n}, \ldots, P_{t, 1} \bullet \cdots \bullet P_{t, n}\right)
$$

of $\mathbb{P}^{n} \times \cdots \times \mathbb{P}^{n}$ is given by the vector space $\Lambda_{F}^{k}(V)$ supported on the $F$-rational point

$$
y^{k}=\left(P_{1,1}^{\bullet k} \bullet \cdots \bullet P_{1, n}^{\bullet k}, \ldots, P_{t, 1}^{\bullet k} \bullet \cdots \bullet P_{t, n}^{\bullet k}\right)
$$

of $\mathbb{P}^{n k} \times \cdots \times \mathbb{P}^{n k}$. (The exponent $\bullet k$ signifies that the point appears $k$ times.) Such objects give a generating set of $K_{0}\left(F, \mathbb{P}^{n} \times \cdots \times \mathbb{P}^{n}\right)$. From this we conclude that the graded lambda ring $K_{0}\left(F, S \cdot \mathbb{P}^{1} \times \cdots \times S \cdot \mathbb{P}^{1}\right)$ is the polynomial ring with coefficients in $\mathbb{Z}$ and with generators given by the set $\mathbb{P}^{1}(F)^{\times t}$ such that the action of $\lambda^{k}$ is determined uniquely by the condition that monomials have rank 1 . As mentioned previously, such a $\lambda$-ring is special and the associated Adams operation $\Psi^{k}$ is the ring map which raises monomials to the $k^{\text {th }}$ power.

Observe that a rational point $p$ of $Y$ determines a map $K_{q}(F) \stackrel{p}{\longrightarrow} K_{q}(F, Y)$ in the evident manner. Using the fact that the diagram

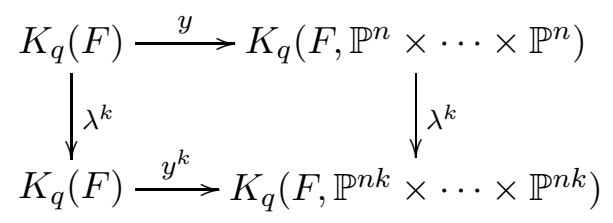

commutes, the remaining claims of the lemma follow. 
Let $F$ be an algebraically closed field. Recall that we use $\gamma_{k}$ to define a map from $K_{q}\left(F, \mathbb{P}^{k} \times \cdots \times \mathbb{P}^{k}\right)$ to $K_{q}\left(F, \mathbb{P}^{1} \times \cdots \times \mathbb{P}^{1}\right)$. It is straightforward to check that when $q=0$, this map takes a $t$-tuple of unordered $n$-tuples of points in $\mathbb{P}^{1}(F)$ and sends it to the sum of all $k^{t}$ possible $t$-tuples of points in $\mathbb{P}^{1}(F)$. The composite map

$K_{0}\left(F, \mathbb{P}^{1} \times \cdots \times \mathbb{P}^{1}\right) \stackrel{\Psi^{k}}{\longrightarrow} K_{0}\left(F, \mathbb{P}^{k} \times \cdots \times \mathbb{P}^{k}\right) \longrightarrow K_{0}\left(R, \mathbb{P}^{1} \times \cdots \times \mathbb{P}^{1}\right)$

is thus multiplication by $k^{t}$. In light of Lemma 7.2, it follows that the Adams operation $\Psi^{k}$ on $K_{q}\left(\mathbb{A}_{k}^{n}, \mathbb{P}^{\wedge t}\right)_{\mathbb{Q}}$ is compatible under the inclusion to $K_{q}\left(\bar{E}, \mathbb{P}^{\wedge t}\right) \cong A \otimes_{\mathbb{Q}} K_{q}(\bar{E})$, where $A$ is a quotient of a polynomial ring, with the map given by $k^{t} \otimes \Psi^{k}$.

LEMMA 7.3. For $F$ a field, the rational homotopy groups $\pi_{n} W^{t}(F)$ lie in $A \mathbb{Q}$.

Proof. Observe that $\Psi^{k}$ acts on the spectral sequence

$$
E_{p, q}^{2}=\pi_{p}\left|d \mapsto K_{q}\left(\Delta_{F}^{d}, \mathbb{P}^{\wedge t}\right)\right| \Longrightarrow \pi_{p+q} W^{t}(F),
$$

since the Adams operations where defined degreewise on the simplicial spaces involved. The $E^{\infty}$-terms are the quotients of a finite filtration of $\pi_{p+q} W^{t}(F)$ and $\Psi^{k}$ acts on this filtration. From Lemma 7.2, we know that the $E^{2}$-terms (after tensoring with $\mathbb{Q}$ ) are objects of $A \mathbb{Q}$, since they arise from special $\lambda$-rings with locally nilpotent gamma filtrations. It follows from Lemma 7.1 that the $E^{\infty}$ terms as well as the abutment are also members of $A \mathbb{Q}$. 
The spectral sequence used in the proof of this lemma provides us a method for understanding the weights of $W^{t}(R)$. Taking generalized eigenspaces is exact on the category $A \mathbb{Q}$ and so tensoring by $\mathbb{Q}$ and then taking weight $t$ parts of (19) yields the spectral sequence

$$
E_{p, q}^{2}=\pi_{p}\left|d \mapsto K_{q}\left(\Delta_{F}^{d}, \mathbb{P}^{\wedge t}\right)\right|_{\mathbb{Q}}^{(i)} \Longrightarrow \pi_{p+q} W^{t}(F)_{\mathbb{Q}}^{(i)}
$$

We can say something nontrivial about the $E_{2}$-terms of this spectral sequence.

PROPOSITION 7.4. For any field $F$, the vector space

$$
\pi_{p}\left|d \mapsto K_{q}\left(\Delta_{F}^{d}, \mathbb{P}^{\wedge t}\right)\right|_{\mathbb{Q}}
$$

is pure and all its weights lie in the set

$$
\{t+\epsilon, t+\epsilon+1, \ldots, t+q\}
$$

where $\epsilon=\min (2, q)$. In particular, if $q \leq 2$, then this space is pure of weight $q+t$.

Proof. In fact, each group $K_{q}\left(\Delta_{F}^{d}, \mathbb{P}^{\wedge t}\right)_{\mathbb{Q}}$ is pure with weights in the indicated set. For by Lemma 7.2 and the discussion following, this vector space injects into $K_{q}\left(\bar{E}, \mathbb{P}^{\wedge t}\right)_{\mathbb{Q}} \cong A \otimes_{\mathbb{Q}} K_{q}(\bar{E})_{\mathbb{Q}}$, where $A$ is a certain quotient of a ring with Adams operation given as multiplication by $k^{t}$. The weights of $K_{q}(\bar{E})_{\mathbb{Q}}$ are known to lie in the set $\{\epsilon, \ldots, \epsilon+q\}$ [14, Corollaire 1 of Théorème 1], and the proposition follows. 
COROLLARY 7.5. For any field $F$, the weight $t$ part of $\pi_{q} W^{t}(F)_{\mathbb{Q}}$ is isomorphic to $K_{q}(F)_{\mathbb{Q}}^{(t)}$.

Proof. Using the spectral sequence (20) and Lemma 7.4, we identify the weight $t$ part of $\pi_{q} W^{t}(F)_{\mathbb{Q}}$ with $\pi_{q}\left|d \mapsto K_{0}\left(\operatorname{Spec} F \times \Delta^{d}, \mathbb{P}^{\wedge t}\right)\right|_{\mathbb{Q}}$. Using a linear version of the Gersten conjecture (see [19, Theorem 3.8]), the group $K_{0}^{\prime}\left(\operatorname{Spec} F \times \Delta^{d}, Y\right)$ is isomorphic to $C_{0}\left(\operatorname{Spec} F \times \Delta^{d}, Y\right)$ for any scheme $Y$. But combining results of Suslin [15, Thoerem 2.1] and Voevodsky [18, Proposition 4.2.9] gives us that the natural inclusion of chain complexes

$$
C_{0}\left(\operatorname{Spec} F \times \Delta^{\bullet}, Y\right) \longrightarrow \mathcal{Z}^{t}(Y, \cdot)
$$

where $\mathcal{Z}^{t}(Y, \cdot)$ is the complex defining Bloch's higher Chow groups, is a quasi-isomorphism whenever $Y$ is an equidimensional projective variety over $F$ of dimension $t$. Applying this to the scheme $\mathbb{P}^{i}$, we get that $C_{0}\left(\operatorname{Spec} F \times \Delta^{\bullet}, \mathbb{P}^{i}\right) \longrightarrow \mathcal{Z}^{i}\left(\mathbb{P}_{F}^{i}, \cdot\right)$ is a quasi-isomorphism. Using the behavior of the Chow groups with respect to projective space [2], it follows $H_{i}\left(C_{0}\left(\operatorname{Spec} F \times \Delta^{\bullet}, \mathbb{P}^{\wedge t}\right)\right) \cong C H^{t}(\operatorname{Spec} F, i)$. By Bloch [2],[3] and also Levine [12] the rational higher Chow groups are rationally isomorphic to $K_{i}(F)^{(t)}$, from which the result follows.

Remark 7.6. The reader should be warned that the proof of the corollary does not show that the map $\pi_{q} W^{t}(F)_{\mathbb{Q}} \longrightarrow K_{q}(F)_{\mathbb{Q}}$ induces 
an isomorphism on weight $t$ parts, although we strongly suspect that this is the case.

We now proceed to show that the filtration $W^{t}(R)$ of $K(R)$ induces that gamma filtration on $K_{q}(R)_{\mathbb{Q}}$. In a sense, all of the work up to this point will not be needed in the sequel, even though we will be employing Adams operations on the homotopy groups of $W^{0 / t}(R)$ (which will be defined shortly).

We say a field $k$ admits resolutions of singularities if Definition 3.4 of [4] is satisfied. The details of this definition are not important in that we require the hypothesis only to apply certain results of [4]. Not surprisingly, if $k$ has characteristic zero, then $k$ admits resolutions of singularities.

THEOREM 7.7. Let $Y$ be a smooth variety over a field $k$ which admits resolution of singularities. Then the map $\pi_{q} W^{t}(Y)_{\mathbb{Q}} \longrightarrow K_{q}(Y)_{\mathbb{Q}}^{(t)}$ is surjective and the map $\pi_{q} W^{t}(Y)_{\mathbb{Q}} \longrightarrow K_{q}(Y)_{\mathbb{Q}}^{(i)}$ is zero for $i<q$. Thus, the filtration on $K_{q}(Y)_{\mathbb{Q}}$ defined by the images of the maps $\pi_{q} W^{t}(Y) \longrightarrow K_{q}(Y)$ is the gamma filtration.

Proof. Recall that $\mathbb{P}^{\wedge t}$ is the cube of schemes which assigns to a subset $I$ of $\{1, \ldots, t\}$ the scheme $\mathbb{P}_{I}^{\wedge t}:=X_{1} \times_{k} \cdots \times_{k} X_{t}$, where

$$
X_{i}= \begin{cases}\mathbb{P}_{k}^{1}, & \text { if } i \in I \text { and } \\ \{1\}, & \text { else. }\end{cases}
$$


For an integer $d$, let $\mathcal{D}_{d}$ denote the collection of $t$-tuples of effective divisors $\left(D_{1}, \ldots, D_{t}\right)$ of $Y \times \Delta^{d} \times \mathbb{P}^{1}$ which are finite over $Y \times \Delta^{d}$ and such that $D_{i}$ contains $Y \times \Delta^{d} \times\{1\}$. For $D \in \mathcal{D}_{d}$ and $I \subset\{1, \ldots, t\}$, let $D_{I}$ denote the closed subscheme of $Y \times \Delta^{d} \times \mathbb{P}_{I}^{\wedge t}$ defined by

$$
E_{1} \times_{Y \times \Delta^{d}} \cdots \times_{Y \times \Delta^{d}} E_{t}
$$

where

$$
E_{i}= \begin{cases}D_{i}, & \text { if } i \in I \text { and } \\ \{1\}, & \text { else. }\end{cases}
$$

For the purposes of this proof we will be modifying our definition of $K\left(Y \times \Delta^{d}, \mathbb{P}_{I}^{\wedge t}\right)$ to be the limit over all $D \in \mathcal{D}_{d}$ of $K$-theory with supports on $D$. First, we define

$$
\begin{aligned}
& K^{D_{I}}\left(Y \times \mathbb{P}_{I}^{\wedge t}\right):= \\
& \quad \text { homotopy fiber }\left(K\left(Y \times \Delta^{d} \times \mathbb{P}_{I}^{\wedge t}\right) \longrightarrow K\left(Y \times \Delta^{d} \times \mathbb{P}_{I}^{\wedge t}-D_{I}\right)\right),
\end{aligned}
$$

and then use the following fact.

LEMMA 7.8. There is a weak equivalence

$$
K\left(Y \times \Delta^{d}, \mathbb{P}_{I}^{\wedge t}\right) \stackrel{\sim}{\longrightarrow} \lim _{\longrightarrow} K^{D_{I}}\left(Y \times \Delta^{d} \times \mathbb{P}_{I}^{\wedge t}\right)
$$

for each fixed $d$.

Proof of Lemma. It suffice to prove this in the case $I=\{1, \ldots, t\}$ and $d=0$. Let $D$ refer ambiguously to both the $t$-tuple $\left(D_{1}, \ldots, D_{t}\right)$ 
and the associated subscheme $D_{1} \times_{Y} \cdots \times_{Y} D_{t}$. Observe that $D$ is clearly a local complete intersection in $Y \times\left(\mathbb{P}^{1}\right)^{\times t}$ and recall $D_{i}$ is necessarily flat over $Y$ by the local criterion of flatness. Using Thomason [17, 5.7], we see that a model for the spectrum $K^{D}\left(Y \times\left(\mathbb{P}^{1}\right)^{\times t}\right)$ is obtained by considering the category of coherent sheaves $\mathcal{F}$ on $Y \times\left(\mathbb{P}^{1}\right)^{\times t}$ which vanish on $Y \times\left(\mathbb{P}^{1}\right)^{\times t}-D$ and admit a resolution of length $t$ by vector bundles. Let us call this category $\mathbb{H}\left(Y \times\left(\mathbb{P}^{1}\right)^{\times t}, D\right)$. Letting $D$ vary and taking unions of the categories $\mathbb{H}\left(Y \times\left(\mathbb{P}^{1}\right)^{\times t}, D\right)$, we obtain a full subcategory of the category of coherent sheaves on $Y \times\left(\mathbb{P}^{1}\right)^{\times t}$, which we will write simply as $\mathbb{H}(Y, t)$. We show the categories $\mathbb{H}(Y, t)$ and $\mathcal{P}\left(Y,\left(\mathbb{P}^{1}\right)^{\times t}\right)$ are actually equal. Given $\mathcal{F} \in \mathbb{H}\left(Y \times\left(\mathbb{P}^{1}\right)^{\times t}, D\right)$, observe that the support of $\mathcal{F}$ is contained $n D$, for $n$ sufficiently large, where $n D$ is defined by $\left(n D_{1}, \ldots, n D_{t}\right)$. In particular, since each $D_{i}$ is finite over $Y$, the support of $\mathcal{F}$ is finite over $Y$. It remains to show the pushforward of $\mathcal{F}$ to $Y$ is locally free. We can reduce to the following local assertion: If $R \longrightarrow S$ is a smooth ring map of relative dimension $t, f_{1}, \ldots, f_{t}$ form a regular sequence in $S$ such that $R \longrightarrow S /\left(f_{1}, \ldots, f_{t}\right)$ is flat, and $M$ is a finitely generated $S$-module of projective dimension at most $t$ such that $f_{i} \cdot M=0$ for all $i$, then $M$ is a flat $R$-module. To show

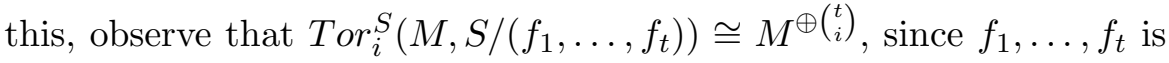


a regular sequence annihilating $M$. Let

$$
0 \longrightarrow P_{t} \longrightarrow \ldots \longrightarrow P_{0} \longrightarrow M \longrightarrow 0
$$

be an $S$-projective resolution of $M$. Tensor this sequence by $S /\left(f_{1}, \ldots, f_{t}\right)$ and restrict scalars to obtain a complex of flat $R$-modules

$$
\ldots \longrightarrow 0 \longrightarrow F_{t} \longrightarrow \ldots \longrightarrow F_{0} \longrightarrow 0 \longrightarrow \ldots
$$

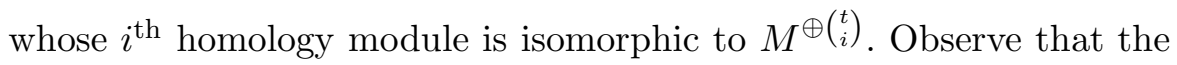
Tor-dimension of $M$ as an $R$-module is finite, since $R \longrightarrow S$ is smooth. Using the complex (21), one easily argues that if $\operatorname{Tor}_{-} \operatorname{dim}_{R} M>0$, then Tor- $\operatorname{dim}_{R} M<\operatorname{Tor}-\operatorname{dim}_{R} M$, a contradiction. Thus, the pushforward of $\mathcal{F}$ to $Y$ is locally free.

Conversely, if $\mathcal{F}$ is an object of $\mathcal{P}\left(Y,\left(\mathbb{P}^{1}\right)^{\times t}\right)$ then its support is a closed subscheme of $Y \times\left(\mathbb{P}^{1}\right)^{\times t}$ which is finite over $Y$. Consider one of the $t$ projections $p: Y \times\left(\mathbb{P}^{1}\right)^{\times t} \longrightarrow Y \times \mathbb{P}^{1}$. Observe $p_{*} \mathcal{F}$ is an object of $\mathcal{P}\left(Y, \mathbb{P}^{1}\right)$. As we showed in Section 2 , a closed subscheme of $Y \times \mathbb{P}^{1}$ finite over $Y$ is necessarily contained in an effective relative divisor provided $Y$ is quasi-projective. Let $D_{i}$ be an effective relative divisor of $Y \times \mathbb{P}^{1}$ over $Y$ containing the support of the direct image of $\mathcal{F}$ under the $i^{\text {th }}$ projection. Then $\mathcal{F}$ is supported on $D_{1} \times_{Y} \cdots \times_{Y} D_{t}$, and it remains to show $\mathcal{F}$ admits a resolution of length $t$ by locally free sheaves. Since $Y$ is quasi-projective, it is enough to show $\mathcal{F}$ has Tor-dimension at most $t$ locally on $Y \times\left(\mathbb{P}^{1}\right)^{\times t}$. Let $x$ be a closed point of $Y \times\left(\mathbb{P}^{1}\right)^{\times t}$ mapping 
to $y \in Y$ under the canonical projection $\pi$. Let $\mathcal{O}_{x}$ and $\mathcal{O}_{y}$ denote the structure sheaves of $x$ and $y$ regarded as closed subschemes. Then we have a spectral sequence

$$
\mathcal{T} \operatorname{or}_{p}^{\pi^{-1}(y)}\left(\mathcal{O}_{x}, \mathcal{T} \operatorname{or}_{q}^{Y \times\left(\mathbb{P}^{1}\right)^{\times t}}\left(\mathcal{O}_{\pi^{-1}(y)}, \mathcal{F}\right)\right) \Longrightarrow \mathcal{T} \operatorname{or}_{p+q}^{Y \times\left(\mathbb{P}^{1}\right)^{\times t}}\left(\mathcal{O}_{x}, \mathcal{F}\right)
$$

which shows $\mathcal{T}$ or ${ }_{n}^{Y \times\left(\mathbb{P}^{1}\right)^{\times t}}\left(\mathcal{O}_{x}, \mathcal{F}\right)=0$ for $n>t$, since we have

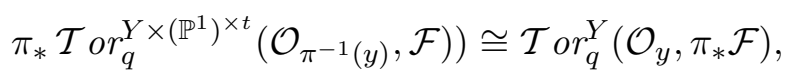

$\pi_{*} \mathcal{F}$ is locally free, and $\pi^{-1}(y)$ is a smooth variety of dimension $t$ over $k(y)$. This completes the proof of the lemma.

The lemma implies that for all $I$ and $d$, there is a fibration sequence $K\left(Y \times \Delta^{d}, \mathbb{P}_{I}^{\wedge t}\right) \longrightarrow K\left(Y \times \Delta^{d} \times \mathbb{P}_{I}^{\wedge t}\right) \longrightarrow \varliminf_{\lim D \in \mathcal{D}_{d}} K\left(Y \times \Delta^{d} \times \mathbb{P}_{I}^{\wedge t}-D_{I}\right)$

Observe that there is an isomorphism $K_{q}\left(Y \times \mathbb{P}^{\wedge t}\right) \cong K_{q}(Y)$ induced by pulling pack to $Y \times\{Q\}$, where $Q$ is a rational point of $\left(\mathbb{P}^{1}\right)^{\times t}$. By an easy special case of the Riemann-Roch theorem, this isomorphism induces an isomorphism

$$
\operatorname{Tot}\left(I \mapsto K_{q}\left(Y \times \Delta^{d} \times \mathbb{P}_{I}^{\wedge t}\right)_{\mathbb{Q}}^{(i-t+|I|)}\right) \cong K_{q}\left(Y \times \Delta^{d}\right)_{\mathbb{Q}}^{(i)}
$$

where we define the total space of a split cube of abelian groups as the iterated cokernel and $|I|$ is the number of elements in $I$. Also by the Riemann-Roch theorem (see, for example, the proof of [12, Lemma 
$2.2])$, we have that $K_{q}^{D_{I}}\left(Y \times \mathbb{P}_{I}^{\wedge t}\right)_{\mathbb{Q}}^{(i)}=0$ for $i<|I|$, since the codimension of $D_{I}$ in $\mathbb{P}_{I}^{\wedge t}$ is $|I|$. It follows that $\pi_{q} W^{t}(Y)_{\mathbb{Q}}^{(i)}$ vanishes for $i<t$, from which the latter claim of the Theorem follows. (Here we are using the induced weight filtration on a homotopy fiber, which is not necessarily the same as that introduced earlier in this paper.)

To prove the theorem, it suffices to show for each fixed $I$, that the image of the map

$$
\pi_{n} K\left(Y \times \Delta^{\bullet}, \mathbb{P}_{I}^{\wedge t}\right)_{\mathbb{Q}} \longrightarrow \pi_{n} K\left(Y \times \Delta^{\bullet} \times \mathbb{P}_{I}^{\wedge t}\right)_{\mathbb{Q}}
$$

contains the weight $|I|$ part. Without loss of generality, we may take $|I|=t$ so that $\mathbb{P}_{I}^{\wedge t}=\left(\mathbb{P}^{1}\right)^{\times t}$. For this to hold, it suffices to show that the weight $t$ part of

$$
\pi_{n}\left(d \mapsto \lim _{\longrightarrow} D \in \mathcal{D}_{d} K\left(Y \times \Delta^{d} \times\left(\mathbb{P}^{1}\right)^{\times t}-D\right)_{\mathbb{Q}}\right)
$$

is zero, where we let $D$ denote ambiguously both a $t$-tuple of divisors $\left(D_{1}, \ldots, D_{t}\right)$ and the closed subscheme $D_{1} \times_{Y \times \Delta^{d}} \cdots \times_{Y \times \Delta^{d}} D_{t}$. Here we are referring to the usual notion of Adams operations on the $K$ theory of a scheme. Technically, what we have here is a simplicial space obtained by taking the limit of the $K$-theory spaces over cosimplicial schemes, and we need that the Adams operations are defined degreewise on the simplicial spaces involved. These issues are addressed in [11]. In particular, the spectral sequence $\pi_{q}\left|d \mapsto \lim _{D \in \mathcal{D}_{d}} K_{p}\left(Y \times \Delta^{d} \times\left(\mathbb{P}^{1}\right)^{\times t}-D\right)\right| \Longrightarrow \lim _{D \in \mathcal{D}_{d}} \pi_{p+q} K\left(Y \times \Delta^{d} \times\left(\mathbb{P}^{1}\right)^{\times t}-D\right)$ 
is compatible with the Adams operations, and we see that we need only show that

$$
d \mapsto \lim _{\longrightarrow} D \in \mathcal{D}_{d} K_{q}\left(Y \times \Delta^{d} \times\left(\mathbb{P}^{1}\right)^{\times t}-D\right)_{\mathbb{Q}}^{(t)}
$$

is acyclic.

By Bloch [2], [3] or Levine [12], the group $K_{q}(W)_{\mathbb{Q}}^{(i)}$ is isomorphic to the rationalized higher Chow group $C H^{q}(W, i)_{\mathbb{Q}}$, for any quasiprojective $W$. When $W$ is smooth, the higher Chow groups can by described be using the family of functors $\mathrm{z}_{\text {equi }}(-, n)(-)$ defined in [4]. In general, for a connected smooth scheme $X$ and an arbitrary scheme $Y$, one defines $\mathrm{z}_{\text {equi }}(Y, n)(X)$ to be the free abelian group on the set of closed integral subschemes of $X \times Y$ which are equidimensional of relative dimension $n$ over $X$ under the evident projection. An extremely important property of this functor is that it satisfies "duality" in the sense that when $X$ is smooth and connected (or just equidimensional), there is a weak equivalence of simplicial abelian groups

$$
e \mapsto \mathrm{z}_{\text {equi }}(Y, n)\left(X \times \Delta^{e}\right) \stackrel{\sim}{\longrightarrow} e \mapsto \mathrm{z}_{\text {equi }}(Y \times X, n+\operatorname{dim} X)\left(\Delta^{e}\right),
$$

induced by the evident inclusion [4, Theorems 8.1 and 8.2 ].

Additionally, the functor $\mathrm{z}_{\text {equi }}\left(\mathbb{P}^{\wedge t}, 0\right)\left(-\times \Delta^{\bullet}\right)$ from smooth schemes to chain complexes of abelian groups (obtained by taking the total complex associated to a $t$-cube of simplicial abelian groups) gives the codi- 
mension $t$ higher Chow groups after taking homotopy groups. (This fact is somewhat buried in the literature, but can be derived by combining Proposition 4.2.9 of [18] with Theorems 8.1 and 8.3 of [4]. Ultimately, it is a consequence of the main result of [15].) Thus the theorem will be proven provided we can show that the $t$-cube of bisimplicial $\mathbb{Q}$-vector spaces

$$
d, e, I \mapsto \lim _{\longrightarrow} D \in \mathcal{D}_{d} \mathrm{z}_{\text {equi }}\left(\mathbb{P}_{I}^{\wedge t}, 0\right)\left(\left(Y \times \Delta^{d} \times\left(\mathbb{P}^{1}\right)^{\times t}-D\right) \times \Delta^{e}\right)_{\mathbb{Q}}
$$

is acyclic; i.e., the associated multidimensional chain complex is acyclic. In fact, we show this integrally, without tensoring with $\mathbb{Q}$.

Let $\left(\mathbb{P}^{1}\right)_{\square}^{\times t}$ refer to the $t$-cube of schemes which has the scheme $\left(\mathbb{P}^{1}\right)^{\times t}$ at the "final" vertex (i.e., the vertex associated to $\{1, \ldots, t\}$ ) and the empty scheme at all other vertices. The central point is the following lemma.

LEMMA 7.9. For $Y$ a smooth, equidimensional, quasi-projective scheme over a field that admits resolution of singularities, the sequence of $t$ cubes of bisimplicial abelian groups

$$
\begin{gathered}
d, e, I \mapsto \mathrm{z}_{\text {equi }}\left(\left(\mathbb{P}^{1}\right)_{\square}^{\times t}{ }_{I}, 0\right)\left(Y \times \Delta^{d}\right) \\
d, e, I \mapsto \mathrm{z}_{\text {equi }}\left(\mathbb{P}_{I}^{\wedge t}, 0\right)\left(Y \times \Delta^{d} \times\left(\mathbb{P}^{1}\right)^{\times t} \times \Delta^{e}\right) \\
d, e, I \mapsto \varliminf_{\lim _{\longrightarrow}} D \in \mathcal{D}_{d} \mathrm{z}_{\text {equi }}\left(\mathbb{P}_{I}^{\wedge t}, 0\right)\left(\left(Y \times \Delta^{d} \times\left(\mathbb{P}^{1}\right)^{\times t}-D\right) \times \Delta^{e}\right)
\end{gathered}
$$


is acyclic; i.e., the associated sequence of multidimensional chain complexes is weakly equivalent to a short exact sequence.

Proof. We may assume $Y$ is connected for simplicity. It suffices to show the sequences of $t$-cubes of simplicial abelian groups obtained by fixing a $d$ and an $I$ are acyclic. Without loss of generality, we may assume $d=0$. Let $y$ be the dimension of $Y$. Then using duality and $[4$, Theorem 8.1 and Corollary 5.12], in the diagram

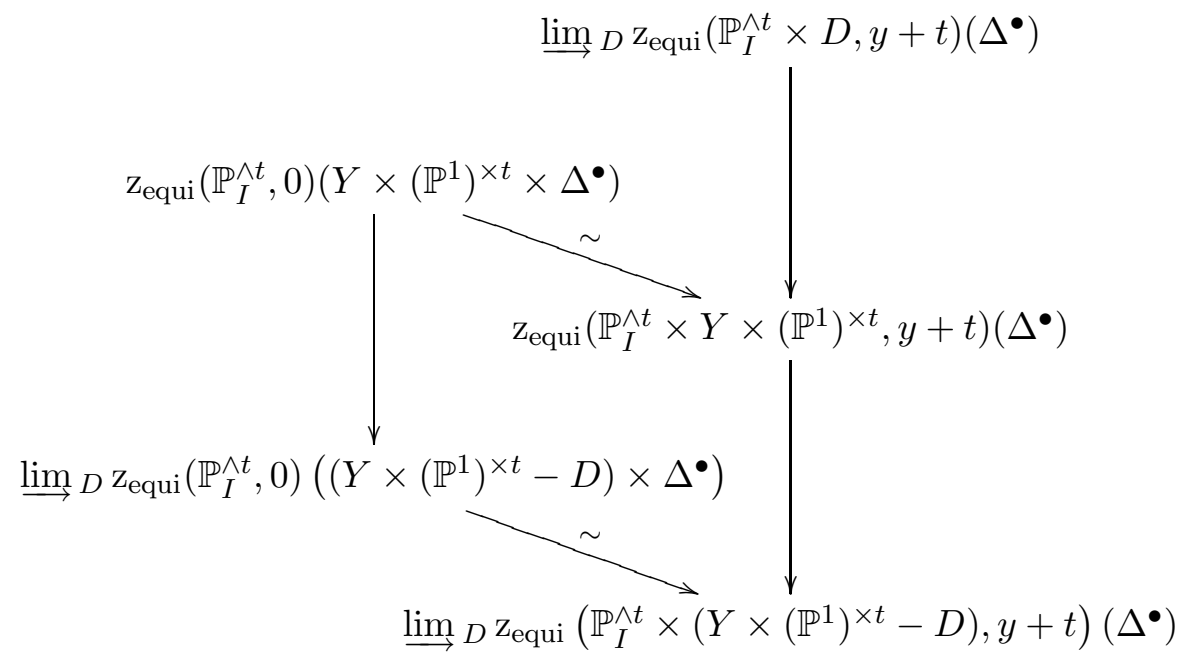

the diagonal arrows are weak equivalences and the right column is acyclic. For $|I|<t$, the top right simplicial abelian group is zero, since $\operatorname{dim} \mathbb{P}_{I}^{\wedge t} \times D<y+t$. It remains to check the case when $|I|=t$. But then $\operatorname{dim} \mathbb{P}_{I}^{\wedge t} \times D=y+t$ for all $D$, and one has that the functor

$$
\mathrm{z}_{\text {equi }}\left(\mathbb{P}_{I}^{\wedge t} \times D, y+t\right)(-)
$$

assigns to any smooth, connected scheme the collection of reduced, irreducible components of $\left(\mathbb{P}^{1}\right)^{\times t} \times D$. Since every closed, integral sub- 
scheme of $Y \times\left(\mathbb{P}^{1}\right)^{\times t}$ which is finite and surjective over $Y$ is contained in some $D$, we have that

$$
\lim _{\longrightarrow} \mathrm{Z}_{\text {equi }}\left(\mathbb{P}_{I}^{\wedge t} \times D, y+t\right)(-)
$$

is the constant functor that assigns to any smooth, connected scheme the abelian group $\mathrm{z}_{\text {equi }}\left(\left(\mathbb{P}^{1}\right)^{\times t}, 0\right)(Y)$. Using the fact that the endomorphism of $\mathrm{z}_{\text {equi }}\left(\left(\mathbb{P}^{1}\right)^{\times t} \times Y \times\left(\mathbb{P}^{1}\right)^{\times t}, y+t\right)\left(\Delta^{\bullet}\right)$ that switches the two copies of $\left(\mathbb{P}^{1}\right)^{\times t}$ is an automorphism, the lemma follows.

Using the lemma, the theorem will hold if the map

$$
\begin{gathered}
d, e, I \mapsto \mathrm{z}_{\text {equi }}\left(\left(\mathbb{P}^{1}\right)_{\square}^{\times t}{ }_{I}, 0\right)\left(Y \times \Delta^{d}\right) \\
\downarrow^{\pi^{*}} \\
d, e, I \mapsto \mathrm{z}_{\text {equi }}\left(\mathbb{P}_{I}^{\wedge t}, 0\right)\left(Y \times \Delta^{d} \times\left(\mathbb{P}^{1}\right)^{\times t} \times \Delta^{e}\right)
\end{gathered}
$$

of $t$-cubes of bisimplicial abelian groups is a weak equivalence. It suffices to show the map of $t$-cubes of simplicial abelian groups obtained by fixing $e$ is a weak equivalence. By homotopy invariance of the homotopy groups of the functor

$$
d, I \mapsto \mathrm{z}_{\text {equi }}\left(\mathbb{P}_{I}^{\wedge t}, 0\right)\left(Y \times \Delta^{d} \times\left(\mathbb{P}^{1}\right)^{\times t} \times-\right),
$$

we may assume $e=0$. By duality, the map

$$
\begin{gathered}
d, I \mapsto \mathrm{z}_{\text {equi }}\left(\mathbb{P}_{I}^{\wedge t}, 0\right)\left(Y \times \Delta^{d} \times\left(\mathbb{P}^{1}\right)^{\times t}\right) \\
\downarrow \\
d, I \mapsto \mathrm{z}_{\text {equi }}\left(\mathbb{P}_{I}^{\wedge t} \times\left(\mathbb{P}^{1}\right)^{\times t}, t\right)\left(Y \times \Delta^{d}\right)
\end{gathered}
$$


is a weak equivalence, since it is for each fixed $I$. Let $\mathbb{A}_{\square}^{t}$ the $t$-cube with $\mathbb{A}^{t}$ concentrated in the final vertex. Then it follows from [4, Corollary 5.12 and Theorem 8.1] that the map of $t$-cubes of simplicial abelian groups

$$
\mathrm{z}_{\text {equi }}\left(\mathbb{P}^{\wedge t} \times\left(\mathbb{P}^{1}\right)^{\times t}, t\right)\left(Y \times \Delta^{\bullet}\right) \longrightarrow \mathrm{z}_{\text {equi }}\left(\mathbb{A}_{\square}^{t} \times\left(\mathbb{P}^{1}\right)^{\times t}, t\right)\left(Y \times \Delta^{\bullet}\right)
$$

is also a weak equivalence. It suffices to show that the composition

$$
\mathrm{z}_{\text {equi }}\left(\left(\mathbb{P}^{1}\right)^{\times t}, 0\right)\left(Y \times \Delta^{\bullet}\right) \longrightarrow \mathrm{z}_{\text {equi }}\left(\mathbb{A}^{t} \times\left(\mathbb{P}^{1}\right)^{\times t}, t\right)\left(Y \times \Delta^{\bullet}\right)
$$

is a weak equivalence. This map clearly factors though $\mathrm{z}_{\text {equi }}\left(\left(\mathbb{P}^{1}\right)^{\times t} \times\right.$ $\left.\left(\mathbb{P}^{1}\right)^{\times t}, t\right)\left(Y \times \Delta^{\bullet}\right)$, and the endomorphism of this simplicial abelian groups that switches the two copies of $\left(\mathbb{P}^{1}\right)^{\times t}$ is obviously an isomorphism. Thus it suffices to show that the map from $\mathrm{z}_{\text {equi }}\left(\left(\mathbb{P}^{1}\right)^{\times t}, 0\right)(Y \times$ $\left.\Delta^{\bullet}\right)$ to $\mathrm{z}_{\text {equi }}\left(\left(\mathbb{P}^{1}\right)^{\times t} \times \mathbb{A}^{t}, t\right)\left(Y \times \Delta^{\bullet}\right)$ given by pulling back along the projection $\left(\mathbb{P}^{1}\right)^{\times t} \times \mathbb{A}^{t} \longrightarrow\left(\mathbb{P}^{1}\right)^{\times t}$ is a weak equivalence. But this map can be factored as

$$
\begin{gathered}
\mathrm{z}_{\text {equi }}\left(\left(\mathbb{P}^{1}\right)^{\times t}, 0\right)\left(Y \times \Delta^{\bullet}\right) \longrightarrow \mathrm{z}_{\text {equi }}\left(\left(\mathbb{P}^{1}\right)^{\times t}, 0\right)\left(Y \times \mathbb{A}^{t} \times \Delta^{\bullet}\right) \longrightarrow \\
\mathrm{z}_{\text {equi }}\left(\left(\mathbb{P}^{1}\right)^{\times t} \times \mathbb{A}^{t}, t\right)\left(Y \times \Delta^{\bullet}\right),
\end{gathered}
$$

and each of these maps is a weak equivalence. The theorem is proven.

Remark 7.10. In the course of the proof, we had occasion to refer to the weight $i$ part of $K_{q}^{D_{I}}\left(Y \times \Delta^{d} \times \mathbb{P}_{I}^{\wedge t}\right)$. This notion of weight uses the 
induced Adams operation on a homotopy fiber of the map $K(X) \longrightarrow$ $K(X-Z), Z$ a closed subscheme of $X$. Taking limits over all $D$, we get Adams operations on $K_{q}\left(Y \times \Delta^{d}, \mathbb{P}_{I}^{\wedge t}\right)$. We do not know if these coincide with the Adams operations introduced earlier in this paper, but we certainly expect that they do. Establishing this agreement would allow one to define Adams operations on Grayson's spectral sequence compatible with the Adams operations on the $K$-groups. We record here a formal statement of this conjecture, which should be viewed as a variation of the Riemann-Roch theorem.

CONJECTURE 7.11. The Adams operations on $\pi_{q} W^{t}(Y)$ defined using the bivariant theory in Section 6 coincide with those defined on the limit of the homotopy fibers of $K\left(Y \times \Delta^{d} \times \mathbb{P}_{I}^{\wedge t}\right) \longrightarrow K\left(Y \times \Delta^{d} \times \mathbb{P}_{I}^{\wedge t}-D_{I}\right)$.

\section{References}

1. M. F. Atiyah and D. O. Tall. Group representations, $\lambda$-rings and the $J$ homomorphism. Topology, 8:253-297, 1969.

2. Specer Bloch. Algebraic cycles and higher K-theory. Adv. Math., 61:267-304, 1986.

3. Spencer Bloch. The moving lemma for higher Chow groups. J. Algebraic Geom., 3:537-568, 1994. 
4. Eric Friedlander and Vladimir Voevodsky. Bivariant cycle cohomology. Preprint.

5. Henri Gillet and Daniel Grayson. On the loop space of the Q-construction. Illinois J. Math., 31:574-597, 1987.

6. Daniel R. Grayson. Algebraic cycles and algebraic K-theory. J. Algebra, 61:129-151, 1979.

7. Daniel R. Grayson. Exterior power operations on higher $K$-theory. $K$-Theory, $3: 247-260,1989$

8. Daniel R. Grayson. Weight filtrations via commuting automorphisms. $K$ theory, 9:139-172, 1995.

9. Howard L. Hiller. $\lambda$-rings and algebraic K-theory. J. Pure Appl. Alg., 20:241266, 1981.

10. Ch. Kratzer. $\lambda$-structure en K-théorie algébrique. Comment. Math. Helv., $55: 233-254,1980$.

11. Marc Levine. Lambda operations, $K$-theory and motivic cohomology. Preprint.

12. Marc Levine. Bloch's higher chow groups revisited. In K-theory, volume 226 of Asterisque, pages 235-320. Société Mathématique de France, 1994.

13. Daniel Quillen. Higher algebraic K-theory: I. In Algebraic K-theory I, volume 341 of Lecture Notes in Math. Springer-Verlag, Berlin, Heidelberg, New York, 1972.

14. Christophe Soulé. Opérations en K-théorie algébrique. Can. J. Math., 37(3):488-550, 1985.

15. A. Suslin. Higher chow groups of affine varieties and étale cohomology. Preprint.

16. A. Suslin and V. Voevodsky. Singular homology of abstract algebraic varieties. Invent. Math., 123:61-94, 1996. 
17. R. W. Thomason and T. Trobaugh. Higher albegraic $K$-theory of schemes and of derived categories. In The Grothendieck Festschrift, Volume III, volume 88 of Progress in Math., pages 247-436. Birkhäuser, Boston, Basél, Berlin, 1990.

18. V. Voevodsky. Triangulated categories of motives over a field. Preprint.

19. Mark E. Walker. Motivic Complexes and the K-theory of Automorphisms. PhD thesis, University of Illinois, Urbana-Champaign, 1996. 